\title{
Arrestin Competition Influences the Kinetics and Variability of the Single-Photon Responses of Mammalian Rod Photoreceptors
}

\author{
Thuy Doan, ${ }^{1}$ Anthony W. Azevedo, ${ }^{2}$ James B. Hurley, ${ }^{1,3}$ and Fred Rieke ${ }^{1,2,4}$ \\ ${ }^{1}$ Program in Neurobiology and Behavior, Departments of ${ }^{2}$ Physiology and Biophysics and ${ }^{3}$ Biochemistry, and ${ }^{4}$ Howard Hughes Medical Institute, \\ University of Washington, Seattle, Washington 98195
}

\begin{abstract}
Reliable signal transduction via G-protein-coupled receptors requires proper receptor inactivation. For example, signals originating from single rhodopsin molecules vary little from one to the next, requiring reproducible inactivation of rhodopsin by phosphorylation and arrestin binding. We determined how reduced concentrations of rhodopsin kinase (GRK1) and/or arrestin1 influenced the kinetics and variability of the single-photon responses of mouse rod photoreceptors. These experiments revealed that arrestin, in addition to its role in quenching the activity of rhodopsin, can tune the kinetics of rhodopsin phosphorylation by competing with GRK1. This competition influenced the variability of the active lifetime of rhodopsin. Biasing the competition in favor of GRK1 revealed that rhodopsin remained active through much of the single-photon response under the conditions of our experiments. This long-lasting rhodopsin activity can explain the characteristic time course of single-photon response variability. Indeed, explaining the late time-to-peak of the variance required an active lifetime of rhodopsin approximately twice that of the G-protein transducin. Competition between arrestins and kinases may be a general means of influencing signals mediated by G-protein-coupled receptors, particularly when activation of a few receptors produces signals of functional importance.
\end{abstract}

\section{Introduction}

G-protein-coupled receptors (GPCRs) regulate numerous biological processes essential for life. These receptors convert extracellular stimuli into intracellular signals by catalyzing G-protein activation. GPCRs regulate our heart rate (Premont and Gainetdinov, 2007), generate the signals that give rise to our sense of smell, taste, and sight (Buck, 2000; Maeda et al., 2003; Chandrashekar et al., 2006; Kobilka and Deupi, 2007), and are the targets of approximately half of modern therapeutic drugs (Kristiansen, 2004; Eglen et al., 2007). Because GPCRs act as catalysts, the sensitivity and reliability of the signals they mediate rely on the regulation of receptor lifetime, yet we lack a quantitative understanding of GPCR inactivation.

Here we investigate the inactivation of rhodopsin, a GPCR that converts the absorption of a single photon into an amplified electrical response in rod photoreceptors (Fig. 1A) (Baylor et al., 1979; Field et al., 2005). A single activated rhodopsin molecule

Received Feb. 18, 2009; revised July 31, 2009; accepted Aug. 2, 2009.

Support was provided by National Institutes of Health Grants EY-11850 (F.R.), T32EY-07031 (T.D.), T32GM07270 (A.W.A), and EY06641 (J.B.H), Poncin Scholarship (T.D.), and the Howard Hughes Medical Institute (F.R.). We are grateful to the following: Lars Holzhausen, Visvanathan Ramamurthy, and Florent Houdart for invaluable help with the protein quantification; Anna Mendez, Jeannie Chen, and Ching-Kang Chen for generously providing Arr ${ }^{-1-}$ and GRK1 ${ }^{-/-}$mice; Ted Wensel for the generous gift of the $\alpha$-RGS9c antibody; Clint Makino for the detailed protocol for experiments using Locke's solutions; Bertil Hille, Peter Detwiler, Charles Asbury, William Zagotta, Ben Pinsky, Jill Jensen, Felice Dunn, and Gabe Murphy for critical reading of this manuscript; the Rieke, Hurley, and Perkel laboratory members for many helpful discussions; and Paul Newman, Richard Alquist, Eric Martinson, and Jonathan Linton (supported through the Vision Core Grant EY01730) for excellent technical support.

Correspondence should be addressed to Fred Rieke, Department of Physiology and Biophysics, University of Washington, 1705 NE Pacific Avenue, Seattle, WA 98195. E-mail: rieke@u.washington.edu.

DOI:10.1523/JNEUROSCI.0819-09.2009

Copyright $\odot 2009$ Society for Neuroscience ～0270-6474/09/2911867-13\$15.00/0
$\left(\mathrm{Rh}^{*}\right)$ catalyzes the activation of multiple copies of the G-protein transducin, which in turn activate phosphodiesterase (PDE) and lead to the closure of cGMP-gated channels in the rod plasma membrane. Amplification persists as long as rhodopsin remains active. As a consequence, the low variability of the single-photon response of the rod requires tight control of the active lifetime of rhodopsin (Rieke and Baylor, 1998; Whitlock and Lamb, 1999; Field and Rieke, 2002a; Hamer et al., 2003).

As with other GPCRs, the lifetime of $\mathrm{Rh}^{\star}$ is controlled by a GPCR kinase (GRK1) and arrestin (arrestin1) (Fig. 1A,B). $\mathrm{Rh}^{*}$ becomes phosphorylated multiple times and then inactivated completely after arrestin1 binding (Wilden and Kühn, 1982; Kühn et al., 1984; Ohguro et al., 1995; Wilden, 1995; Chen et al., 1995, 1999; Xu et al., 1997; Mendez et al., 2000; Kennedy et al., 2001; Maeda et al., 2003; Makino et al., 2003). Rh* phosphorylation involves GRK1 binding to $\mathrm{Rh}^{\star}$ (Fig. $1 \mathrm{~B}$, reaction 2 ) and the attachment of a phosphate group to the $\mathrm{C}$ terminus (reaction 3 ). Arrestin 1 then quenches $\mathrm{Rh}^{\star}$ activity (reaction 4 ) by binding to phosphorylated $\mathrm{Rh}^{\star}$ at multiple sites (Gurevich and Benovic, 1993; Gurevich and Gurevich, 2004). The arrestin binding rate depends on the concentration of arrestin 1 in the rod outer segment, whereas the efficacy of quenching depends on the number of phosphates attached to the $\mathrm{C}$ terminus of $\mathrm{Rh}^{\star}$ (Kühn et al., 1984; Wilden, 1995; Xu et al., 1997; Gibson et al., 2000; Ling et al., 2004; Vishnivetskiy et al., 2007).

In addition to its established role in quenching rhodopsin activity, Figure $1 B$ depicts a new hypothesized role of arrestin 1 : competition with GRK1 for $\mathrm{Rh}^{\star}$ binding sites. Several observations motivate this competition hypothesis. First, GRK1 and arrestin 1 share partially overlapping binding sites on the cytoplasmic domains of rhodopsin (Kelleher and Johnson, 1990; 
Palczewski et al., 1991; Krupnick et al., 1994; Shi et al., 1995; Raman et al., 1999, 2003). Second, both in vitro and in vivo studies show that arrestin1 is capable of high-affinity binding to phosphorylated rhodopsin and low-affinity binding to unphosphorylated rhodopsin (Gurevich and Benovic, 1992, 1995; Krupnick et al., 1994; Raman et al., 1999; Vishnivetskiy et al., 1999; Gurevich and Gurevich, 2004; Burns et al., 2006). Together, these studies suggest that competition between arrestin1 and GRK1 might regulate GRK1 binding to rhodopsin and thus modulate the active lifetime of rhodopsin. Here we test this hypothesis by measuring how alterations in the concentration of GRK1 and/or arrestinl affect the kinetics and variability of the single-photon responses of a rod.

\section{Materials and Methods}

Animals. All animal procedures were approved by the Administrative Panel on Laboratory Animal Care at the University of Washington. Control C57BL/6 mice (5 weeks old) were purchased from Harlan Sprague Dawley. Arr1 ${ }^{-/-}$ and $G R K 1^{-1-}$ mice were obtained from Dr. Jeannie Chen (University of Southern California, Los Angeles, CA). Arr1 ${ }^{+/-}$and $G R K 1^{+/-}$ mice were generated by crossing $A r r 1^{-1-}$ and $G R K 1^{-/-}$with C57BL/6 mice. Some GRK1 ${ }^{+/-}$ mice used for the initial recordings were provided by Dr. Ching-Kang Chen (Virginia Commonwealth University, Richmond, VA). Arr ${ }^{-1-}$ mice were crossed with $G R K 1^{-/-}$to generate double heterozygotes $\left(G R K 1^{+/-}\right.$ Arr ${ }^{+/-}$). Mice were genotyped using PCR primers specific to the mutations.

To control for the possibility that differences in genetic background could produce differences in response kinetics, we compared responses of rods from sibling $\mathrm{Arrl}^{+/-}$ and $\mathrm{Arrl}^{+/+}$mice generated from crosses of $\mathrm{Arrl}^{+/-}$parents. Single-photon responses of $\mathrm{ArrI}^{+/-}$rods were smaller and faster than those of $\mathrm{Arrl}^{+/+}$rods. Data from siblings and nonsiblings were similar and have been pooled in Table 1.

Electrophysiology. Mice were dark adapted overnight and killed by cervical dislocation. The dissection was performed under infrared light $(>900 \mathrm{~nm})$ to keep the retina fully dark adapted. Isolated retinas were stored in a light-tight container at $32^{\circ} \mathrm{C}$ in bicarbonate-buffered Ames solution (Sigma) equilibrated with $5 \% \mathrm{CO}_{2} / 95 \% \mathrm{O}_{2}$. Photoresponses were recorded using suction electrodes (Baylor et al., 1979; Field and Rieke, 2002a; Doan et al., 2006). A small piece of retina was mechanically shredded, transferred into a recording chamber, and perfused at $\sim 3.5$ $\mathrm{ml} / \mathrm{min}$ with bicarbonate-buffered Ames solution heated to $30 \pm 1^{\circ} \mathrm{C}$ or $36 \pm 1^{\circ} \mathrm{C}$. The outer segment currents of single rod photoreceptors were recorded using suction electrodes with optimized geometry. Borosilicate glass capillary tubes were pulled to taper abruptly to an opening of 4-6 $\mu \mathrm{m}$. The tips were polished to an inner diameter $(1.6-2.2 \mu \mathrm{m})$ to encompass snugly the rod outer segment, which was positioned inside the electrode to maximize the dark current. The electrode contained HEPES Ames solution $\left(\mathrm{NaHCO}_{3}\right.$ replaced with $10 \mathrm{~mm}$ HEPES and $15 \mathrm{~mm} \mathrm{NaCl}$, $\mathrm{pH} 7.40$ with $\mathrm{NaOH}$ ). Most studies of retinal circuitry in mammals use similar recording conditions. arrestin1 concentration.
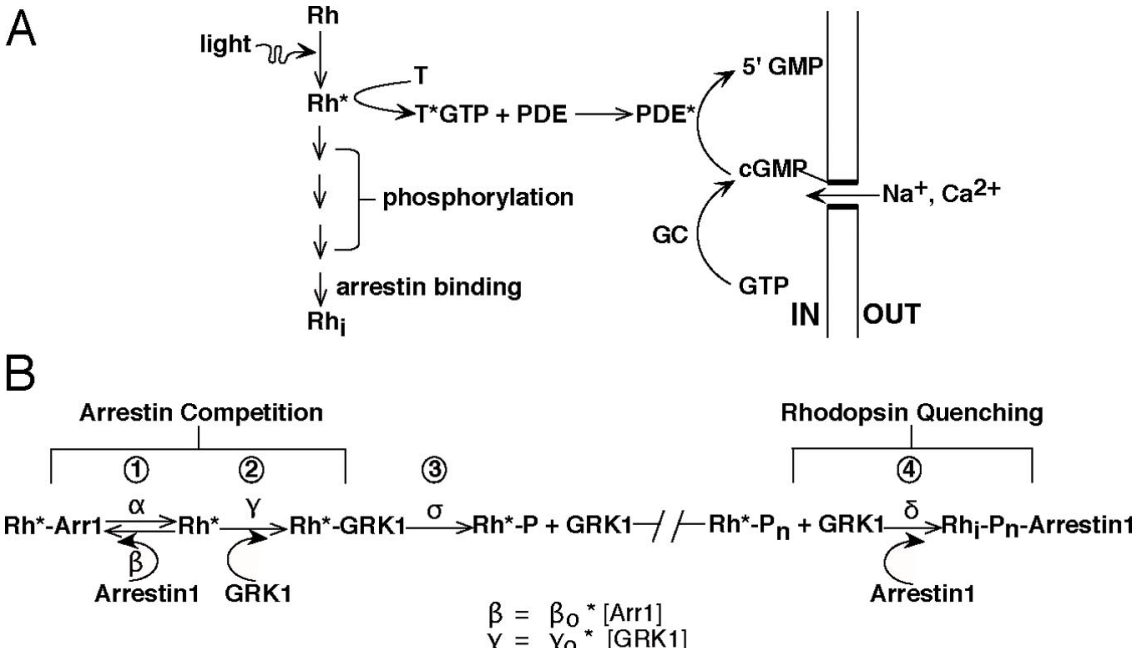
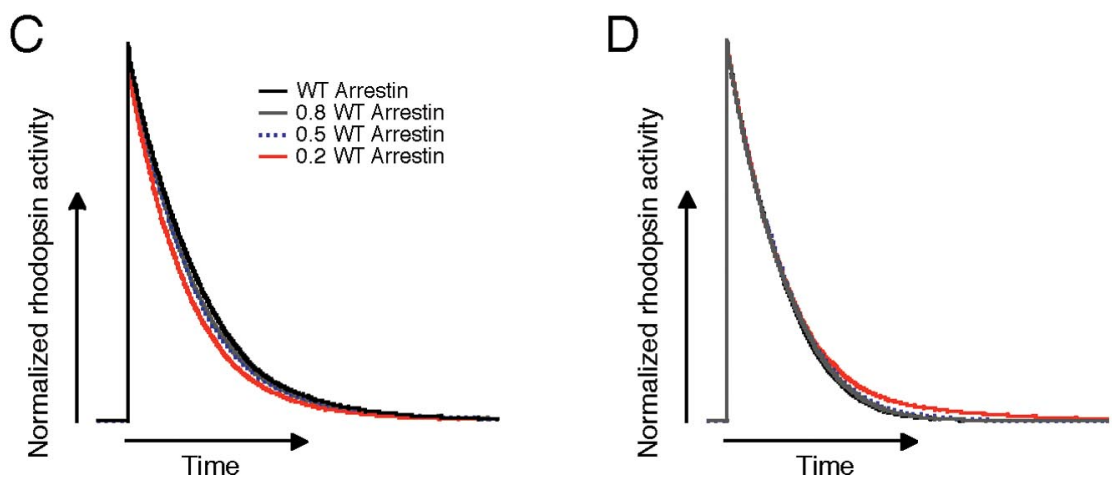

Figure 1. Biochemical readout and control of rhodopsin activity. $\boldsymbol{A}$, Schematic of phototransduction cascade in mammalian ods. Rhodopsin (Rh) is activated by the absorption of a photon. Activated rhodopsin ( $\mathrm{Rh}^{*}$ ) leads to the sequential activation of inactivation of an Rh* molecule. The duration of each rhodopsin phosphorylation event depends on the competition rrestin1 and GRK1 binding, and the time required for phosphate attachment. Reactions 1-3 cycle until a sufficient number of phosphates $\left(P_{n}\right)$ are incorporated to the $C$ terminus. Wild-type mouse rhodopsin has six potential phosphorylation sites. The final $h^{*}-P_{n}$ (reaction 4) terminates $R h^{*}$ activity. C, Simulation of the combined effect of arrestin competition and quenching on

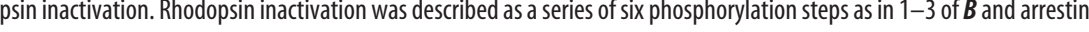
net effect of decreasing arrestin1 concentration can be to shorten $\mathrm{Rh}^{*}$ activity. $\boldsymbol{D}$, Decreasing the arrestin1 concentration would lengthen $\mathrm{Rh}^{*}$ activity if the only role of arrestin is to quench $\mathrm{Rh}^{*}$. Simulations were identical to $C$ except $\beta$ did not depend on

Our recorded rod responses showed systematically slower kinetics and higher sensitivity than other published work (Xu et al., 1997; Mendez et al., 2000; Calvert et al., 2001; Krispel et al., 2006). The main differences in conditions are the solutions used for retina storage and recording. Thus, in a few control experiments, we made the following substitutions: (1) we stored the retina in ice-cold, oxygenated L-15 medium (Invitrogen) mixed with $0.1 \mathrm{mg} / \mathrm{ml} \mathrm{BSA} \mathrm{(Fraction} \mathrm{V;} \mathrm{Sigma)} \mathrm{and} 10 \mathrm{~mm}$ glucose; (2) we perfused the recording chamber with bicarbonate-buffered Locke's solution [112.5 mM NaCl, 3.6 mM KCl, $2.4 \mathrm{~mm} \mathrm{MgCl}$, $1.2 \mathrm{mM} \mathrm{CaCl}_{2}, 20$ mM $\mathrm{NaHCO}_{3}, 10 \mathrm{~mm}$ HEPES, 0.02 mm EDTA, $3 \mathrm{~mm}$ succinate, and 0.5 mM L-glutamate, with $10 \mathrm{~mm}$ glucose, vitamin and amino acid supplements (Sigma), and $0.1 \mathrm{mg} / \mathrm{ml} \mathrm{BSA}$ added on the day of experiment] saturated with $5 \% \mathrm{CO}_{2} / 95 \% \mathrm{O}_{2}$ and heated to $36 \pm 1{ }^{\circ} \mathrm{C}$; and (3) we filled the suction electrode with HEPES-buffered Locke's solution $(130 \mathrm{~mm}$ $\mathrm{NaCl}, 3.6 \mathrm{~mm} \mathrm{KCl}, 2.4 \mathrm{~mm} \mathrm{MgCl}_{2}, 1.2 \mathrm{CaCl}_{2}, 10 \mathrm{~mm}$ HEPES, 0.02 EDTA, $0.02 \mathrm{~mm}$ EDTA, $3 \mathrm{~mm}$ succinate, and $0.5 \mathrm{~mm}$ L-glutamate, with $10 \mathrm{~mm}$ glucose and $0.1 \mathrm{mg} / \mathrm{ml} \mathrm{BSA}$ added on the day of experiment, $\mathrm{pH}$ 7.4). The kinetics and sensitivity of the responses recorded under these conditions 
Table 1. Comparison of response properties of rods with normal and reduced GRK1 and arrestin1 concentrations

\begin{tabular}{|c|c|c|c|c|}
\hline Strain & Single-photon response amplitude (pA) & Integration time (ms) & $\mathrm{CV}_{\text {area }}$ & $t_{\text {peak }, \sigma^{2} / t_{\text {peak }, \mu}}$ \\
\hline \multicolumn{5}{|l|}{ Isolated single-photon responses } \\
\hline Wild type $(n=29)$ & $1.41 \pm 0.09$ & $510 \pm 20$ & $0.34 \pm 0.01$ & $2.00 \pm 0.20$ \\
\hline $\mathrm{GRK}^{+1-}(n=30)$ & $1.61 \pm 0.06$ & $840 \pm 30$ & $0.40 \pm 0.02$ & $2.90 \pm 0.13$ \\
\hline $\mathrm{GRK1}^{+/-} \operatorname{Arr}^{+/-}(n=40)$, & $1.45 \pm 0.05$ & $630 \pm 20$ & $0.43 \pm 0.01$ & $2.60 \pm 0.09$ \\
\hline $\operatorname{Arr}^{+/-}(n=41)$ & $1.30 \pm 0.03$ & $500 \pm 20$ & $0.43 \pm 0.02$ & $1.88 \pm 0.08$ \\
\hline \multicolumn{5}{|l|}{ Dim flashes, $30^{\circ} \mathrm{C}$} \\
\hline Wild type $(n=38)$ & $1.02 \pm 0.03$ & $490 \pm 20$ & & \\
\hline$G R K 1^{+/-}(n=43)$ & $1.30 \pm 0.04$ & $800 \pm 20$ & & \\
\hline $\mathrm{GRK1}^{+/-} \operatorname{Arr}^{+/-}(n=39)$, & $1.11 \pm 0.05$ & $610 \pm 20$ & & \\
\hline $\operatorname{Arr}^{+/-}(n=36)$ & $0.76 \pm 0.03$ & $455 \pm 15$ & & \\
\hline \multicolumn{5}{|l|}{ Dim flashes, $36^{\circ} \mathrm{C}$} \\
\hline Wild type $(n=15)$ & $0.98 \pm 0.02$ & $330 \pm 20$ & & \\
\hline $\operatorname{Arr}^{+\prime-}(n=18)$ & $0.64 \pm 0.01$ & $275 \pm 20$ & & \\
\hline
\end{tabular}

For dim flashes, the amplitude of the mean population single-photon response was determined by dividing the mean response by the number of photoisomerizations, determined by scale factor required to match the time-dependent variance with the square of the mean response. Integration time is the integral of the response normalized by its peak amplitude. $\mathrm{CV}_{\text {area }}$ is the SD of the integral of the single-photon response (corrected for dark noise) divided by the mean.

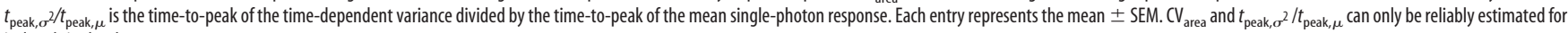
isolated single-photon responses.

differed substantially from our standard conditions (supplemental Fig. 1, available at www.jneurosci.org as supplemental material). Specifically, estimated single-photon responses in L-15/Locke's reached a peak amplitude of $0.2-0.3 \mathrm{pA}$ at a time $110-120 \mathrm{~ms}$ after the flash compared with $1 \mathrm{pA}$ and 190-200 ms in Ames solution. Similarly, Pepperberg time constants (see below) in L-15/Locke's solution were $\sim 200$ ms compared with $\sim 320 \mathrm{~ms}$ in Ames solution. The properties of responses measured in L-15/Locke's solution resembled those reported previously (Xu et al., 1997; Mendez et al., 2000; Calvert et al., 2001; Krispel et al., 2006). The origin of these differences is unclear but will be an important target for future work.

Light stimuli. Light delivered from a light-emitting diode (LED) with peak output at $470 \mathrm{~nm}$ was directed to the cell via a light guide and focused with an $60 \times$ microscope objective $(0.95$ numerical aperture; Nikon). For the Pepperberg time constant experiments, light from the LED was focused to illuminate uniformly a square area of $65 \mu \mathrm{m}$ on a side, centered on the recorded cell. For isolation of single-photon responses, light stimuli were restricted to a slit (width $<1 \mu \mathrm{m}$ ), positioned perpendicular to the long axis of the outer segment approximately halfway along its length. For dim flash response experiments, light from the LED uniformly illuminated a circular area $650 \mu \mathrm{m}$ in diameter centered on the recorded cell. All light stimuli were 10 or $30 \mathrm{~ms}$ in duration. Calibrated light intensities were converted to photon flux (photons per square micrometer per second) at $500 \mathrm{~nm}$ (at which rod sensitivity is maximal) using the measured LED spectral output and the spectral sensitivity function of the rod.

Pepperberg time constant. To estimate the rate of decay of lightactivated PDE activity, we used bright flashes, $30 \mathrm{~ms}$ in duration, to suppress the dark current of the rod for at least $200 \mathrm{~ms}$ and allow $\left[\mathrm{Ca}^{2+}\right]_{i}$ to fall to a minimum. Saturation time was defined as the period over which the flash suppressed $80 \%$ of the dark current (Zhang et al., 2007). Assuming that light-activated PDE activity decays exponentially and that guanylate cyclase activity is maximal and equal for the duration of the saturated response, the slope of the saturation time as a function of the natural $\log$ of the flash strength provides an estimate of the time constant for the decay of PDE activity $\left(\tau_{\mathrm{PDE}}\right)$ (Pepperberg et al., 1992). Because the decay of PDE activity requires both rhodopsin and transducin shutoff, $\tau_{\mathrm{PDE}}$ could reflect the decay time of rhodopsin, transducin, or both.

Dim flash responses. We estimated average single-photon responses from responses to a repeated dim flash. All cells with low baseline noise and a dark current of $>8 \mathrm{pA}$ at $30 \pm 1^{\circ} \mathrm{C}$ and $>12 \mathrm{pA}$ at $36 \pm 1^{\circ} \mathrm{C}$ were retained for this analysis. The mean single-photon response was estimated by dividing the average response to a dim flash by the number of $\mathrm{Rh}^{\star}$ produced by the flash, calculated from the scale factor required to match the first 200-300 ms of the time-dependent variance and the square of the mean response. The estimated collecting areas (conversions between photons per square micrometer and $\mathrm{Rh}^{\star}$ ) were, on average, $\sim 0.5 \mu \mathrm{m}^{2}$ (Field and Rieke, 2002b).
Isolation of single-photon responses. Characterizing variability of the single-photon response requires separating responses to single absorbed photons from those to 0 or multiple photons. Isolation of single-photon responses was more reliable at $30^{\circ} \mathrm{C}$ than at $36^{\circ} \mathrm{C}$, and hence most of our experiments were at $30^{\circ} \mathrm{C}$. We used four criteria to determine whether to collect data from a recorded cell: (1) a dark current exceeding $8 \mathrm{pA}$ at $30 \pm 1{ }^{\circ} \mathrm{C}$; (2) qualitatively low baseline dark noise; (3) discernable singlephoton responses; and (4) stable response kinetics. Approximately $20 \%$ of the recorded cells met all four criteria. From this population of selected cells, $\sim 20-30 \%$ yielded discrete amplitude histograms (see below) and were retained for additional analysis. Recorded currents were low-pass filtered at $30 \mathrm{~Hz}$ (eight-pole Bessel), digitized at $1 \mathrm{kHz}$, and digitally filtered at $5 \mathrm{~Hz}$ before analysis. Periodic checks of response stability followed previous procedures (Field and Rieke, 2002a; Doan et al., 2006).

The procedure used to identify single-photon responses and check for errors in identification has been described in detail previously (Field and Rieke, 2002a). Briefly, the amplitudes of the responses to a repeated dim flash were determined from the vertical scale factor required to fit each response with the average dim-flash response. The fit was restricted to the initial $450-500 \mathrm{~ms}$ of the response, which included the rising phase but not the recovery phase. This procedure was chosen to minimize errors in identifying single-photon responses; all subsequent analysis used the entire response time course.

Histograms of the response amplitudes were fitted assuming that dark noise and noise from the single-photon responses were independent and additive. In this case, the number of responses with an amplitude between $A-\Delta A / 2$ and $A+\Delta A / 2$ is

$$
\begin{aligned}
N(A)=\Delta A \sum_{n=0}^{\infty} \frac{\exp (-\bar{n}) \bar{n}^{n}}{n !}\left[2 \pi\left(\sigma_{D}^{2}+n \sigma_{A}^{2}\right)\right]^{-1 / 2} & \\
& \times \exp \left(-\frac{(A-n \bar{A})^{2}}{2\left(\sigma_{D}^{2}+n \sigma_{A}^{2}\right)}\right),
\end{aligned}
$$

where $\bar{A}$ is the mean single-photon response amplitude and $\sigma_{A}$ is its SD, $n$ is the number of photoisomerizations $\left(\mathrm{Rh}^{*}\right)$ produced by the flash and $\bar{n}$ is its mean, and $\sigma_{\mathrm{D}}$ is $\mathrm{SD}$ of the dark current noise. Thresholds used to identify single-photon responses were set at $3 \bar{A} / 2$ and near $\bar{A} / 2$, or lower if $\sigma_{\mathrm{D}}$ was small. This procedure was repeated for three different flash strengths in each cell. Repeating this procedure at multiple flash strengths allowed the accuracy of the isolation procedure to be determined by checking that the isolated single-photon responses did not depend on the strength of the flash used to elicit them (Field and Rieke, 2002a; Doan et al., 2006).

The isolation procedure described above produced a bias in favor of rods that produced large single-photon responses because small responses could not be reliably distinguished from responses to 0 or multiple photons. For wild-type (WT), GRK1 $1^{+/-}$and $G R K 1^{+/-} A r r 1^{+/-}$ 
cells, this bias was restricted to the response amplitude, whereas both amplitude and kinetics of the responses of $A r r 1^{+/-}$rods were effected (Table 1).

Markov model for single rhodopsin phosphorylation. To provide a quantitative picture of arrestin competition, we implemented a single phosphorylation step in the kinetic scheme of Figure $1 B$ as a Markov chain. We assumed that each phosphorylation step followed the same sequence, i.e., that GRK1 dissociates after phosphate attachment and the cycle starts anew. Two issues determined the ability of arrestin competition to control the rate of phosphorylation: the fraction of time rhodopsin spends bound to arrestin1, and the time required for GRK1 binding compared with that for phosphate attachment. These issues depend on the ratios $\beta / \alpha$ and $\gamma / \sigma$, and hence the goal of the model was to estimate these ratios from the data.

Activated rhodopsin could exist in four states: (1) bound to arrestin $1\left(\mathrm{Rh}^{*}-\mathrm{Arr} 1\right)$; (2) unbound $\left(\mathrm{Rh}^{*}\right)$; (3) bound to GRK1 $\left(\mathrm{Rh}^{*}-\right.$ GRK1); or (4) phosphorylated $\left(\mathrm{Rh}^{\star}-\mathrm{P}\right)$. A state vector $\vec{s}(t)$ captured the probability of each state at time $t$. Every time step $\Delta T, \vec{s}(t)$ was updated using a transition matrix $M$ containing the relevant transition rates from Figure $1 B$, i.e., $\vec{s}(t+\Delta T)=M \times \vec{s}(t)$. This procedure was iterated to determine the mean phosphorylation time (i.e., the time to transition from state 2 to state 4 ) for a given set of rate constants $(\alpha, \beta, \gamma, \sigma)$.

To compare predictions of the Markov model for wild-type and mutant rods, we assumed that the rate constants for arrestin 1 and GRK1 binding to rhodopsin scaled with their concentrations, e.g., $\beta / \beta_{0}=$ $[$ Arr 1$] /[\operatorname{Arr} 1]_{\mathrm{WT}}$, where $\beta_{0}$ is the arrestin 1 association rate in wild-type rods (see below for quantification of protein concentrations). This assumption will hold if the concentration of arrestin1 monomer in the outer segment (the active form) scales linearly with the total arrestin1 concentration. A similar logic applies to the GRK1 concentration. Two issues could effect this assumption. First, a small fraction of arrestin 1 is in the outer segment under dark-adapted conditions (Broekhuyse et al., 1985; Peterson et al., 2003), and this fraction may differ for wild-type, $\mathrm{Arrl}^{+/-}, \mathrm{GRK1}^{+/-}$, and $\mathrm{GRK1}^{+/-} \mathrm{Arrl}^{+/-}$mice (Hanson et al., 2007a). Second, self-association of arrestin 1 will cause the monomer concentration to change less than the total arrestin 1 concentration; the magnitude of this effect ranges from $\sim 20$ to $60 \%$ (Imamoto et al., 2003; Hanson et al., 2007b). Because of the uncertainties associated with these effects, we did not include them in the Markov model. Nonetheless, the central conclusions of the model — that rhodopsin spends most of its time bound to arrestin1 and that GRK1 binding is fast compared with phosphate attachment-held for estimates of the changes in arrestin 1 concentration that included self-association.

Transducin also competes with arrestin1 and GRK1 for rhodopsin binding (Wilden et al., 1986; Krupnick et al., 1997). Thus, a complete description of arrestin competition will need to include its effect on transducin binding. This effect will depend on both the rate constant for transducin binding to free rhodopsin and the rate constant for GDPGTP exchange once transducin has bound. We did not include this effect in the Markov model because of the uncertainties associated with the relevant rate constants. Nonetheless, the impact of arrestin competition on transducin activation is likely to be important and will need to be incorporated in extensions of models like those used here.

Phototransduction cascade model. We used a stochastic model for rhodopsin inactivation (Rieke and Baylor, 1998; Field and Rieke, 2002a; Doan et al., 2006) to investigate how the time-dependent variance of the single-photon response depends on the relative inactivation rates of rhodopsin and transducin. The catalytic activity of rhodopsin was assumed to shut off through a series of phosphorylation events, each composed of two steps (Fig. $1 B$ ): GRK1 binding and phosphate attachment. The simulations assumed a GRK1 binding rate $(\gamma)$ eight times larger than the rate of phosphate attachment $(\sigma)$ (see Results). Each phosphorylation cycle, on average, controlled an equal fraction of the total catalytic activity of rhodopsin; this assumption ensures that multiple phosphorylation cycles contribute maximally to reducing single-photon response variability. This constraint was met by assuming that each step produced an equal reduction in activity (see Fig. $5 C$ ) and that the rate constants for phosphorylation decreased linearly with the number of attached phosphates. Such a progression of rate constants might be produced, at least in part, as rhodopsin is phosphorylated and the affinity of the interaction be- tween arrestin 1 and rhodopsin increases (Wilden, 1995; Gibson et al., 2000; Gurevich and Gurevich, 2004; Vishnivetskiy et al., 2007). This increased affinity should cause arrestin 1 to compete more effectively with GRK1 and hence delay its binding to rhodopsin. Arrestin could terminate the activity of rhodopsin after any number of phosphorylation events, but the rate constant for arrestin quenching increased exponentially with the number of completed phosphorylation cycles (Hamer et al., 2003; Vishnivetskiy et al., 2007).

The time course of the activity of a single simulated rhodopsin molecule was converted to a change in current through either a linear or nonlinear approximation to the behavior of the post-rhodopsin components of the phototransduction cascade. In the linear case, the cascade was approximated by a filter with a Fourier transform given by the following:

$\tilde{F}(\omega)$

$$
=\frac{G_{D}}{[\phi-i \omega]\left[P_{D}+12 \theta^{2} P_{D} /\left(\theta^{2}+\omega^{2}\right)-i \omega\left(1-12 \theta P_{D} /\left(\theta^{2}+\omega^{2}\right)\right)\right]},
$$

where $\omega$ is the temporal frequency in radians per second, $G_{\mathrm{D}}$ is the dark cGMP concentration, $\phi$ is the rate constant for PDE activity, $\theta$ is the rate constant for removal of $\mathrm{Ca}^{2+}$ from the outer segment by $\mathrm{Na}^{+} /$ $\mathrm{K}^{+}, \mathrm{Ca}^{2+}$ exchange, $P_{\mathrm{D}}$ is the dark PDE activity, and $\tilde{F}(\omega)=$ $\int \exp (i \omega t) F(t) d t$ (Rieke and Baylor, 1998). The inverse of $\phi$ is a measure of the average active lifetime of transducin, $\tau_{\mathrm{T}^{*}}$. Equation 1 assumes that the cooperativity of the $\mathrm{Ca}^{2+}$ dependence of guanylate cyclase activity is 4 (Koch and Stryer, 1988; Burns et al., 2002). $G_{\mathrm{D}}$ was determined from the measured dark current, $I_{\mathrm{D}}$, assuming $I_{\mathrm{D}}=k G_{D}^{3}$ with $k=0.0026$ $\mathrm{pA} / \mu \mathrm{M}^{3}$. Modeled single-photon responses used the following parameters: $\theta=10 \mathrm{~s}^{-1}$ (Calvert et al., 2001; Burns et al., 2002), $P_{\mathrm{D}}=1 \mathrm{~s}^{-1}$ (Tamura et al., 1991), and $\phi=5 \mathrm{~s}^{-1}$ (Krispel et al., 2006). In the nonlinear case, the filtered response was passed through a compressive nonlinearity, so that the resulting current was given by the following:

$$
I(t)=1-\exp \left(\int d \tau F(\tau) R(t-\tau) / \eta\right),
$$

where $R$ is the time course of the activity of rhodopsin, and $\eta$ controls the degree of compression. In the absence of compression, the mean current response had a peak amplitude of 1 . Compression reduced the sensitivity of the measured current to variations in the activity of rhodopsin, particularly near the peak of the current response. Such a compression could be produced by local saturation of the transduction cascade (but see Field and Rieke, 2002a for experiments that limit the extent of local saturation).

The behavior of the general model described above is determined by the number of phosphorylation events, the rate of arrestin quenching, and the extent of nonlinear compression. The choice of these model parameters is constrained by the essential properties of the measured single-photon responses: their low variability and the late time-to-peak and the near symmetric shape of the time-dependent variance (Field and Rieke, 2002a). We focused on two models that satisfied these general constraints. In the first, each phosphorylation site contributed equally to rhodopsin shutoff, and, on average, 5.6 phosphorylation cycles completed before rhodopsin was inactivated by arrestin. No compression was required for this model. In the second case, rhodopsin shut off through a maximum of four phosphorylation events, with an average of 3.7 events before arrestin binding. Compression $(\eta=0.6)$ was required to reduce response variability to experimental levels. This model is a simplified version of a comprehensive biophysical model introduced recently (Bisegna et al., 2008) (see also Field and Rieke, 2002a).

In Results, we emphasize the first model above because it has fewer free parameters. However, the central conclusion of the modeling-that the late time-to-peak of the time-dependent variance requires that rhodopsin inactivates more slowly than transducin-held for both models. More generally, this conclusion held for multiple variants of the basic model described above in which each step in rhodopsin inactivation 
decreased its catalytic activity. This included models in which only phosphate attachment, and not GRK1 binding, decreased the catalytic activity of rhodopsin.

Quantification of protein expression. Retinas from wild-type mice, aged 5-8 weeks, and heterozygous knock-out mice, aged 4-6 weeks, were harvested in ice-cold HEPES Ames buffer ( $\mathrm{pH} 7.40)$. Retinas were immediately frozen on dry ice and stored at $-80^{\circ} \mathrm{C}$ until use. Frozen retinas were thawed and homogenized using a handheld homogenizer with PBS/ $0.1 \mathrm{~mm}$ DTT and a mixture of protease inhibitors (Roche Diagnostics). Homogenized tissues were solubilized in $1 \times$ SDS lysis buffer $(62.5 \mathrm{~mm}$ Tris-HCl, pH 6.8, 2\% SDS, $10 \%$ glycerol, $0.005 \%$ bromophenol blue, and $5 \%$ 2-mercaptoethanol) and left at room temperature for $15 \mathrm{~min}$. Retinal equivalent dilutions were separated by $10 \%$ SDS-PAGE, transferred overnight onto nitrocellulose membranes (Millipore Corporation), and then blocked in Odyssey blocking buffer (LI-COR Biosciences).

To quantify GRK1 and arrestin1 expression, the membranes were sequentially incubated with a different set of primary and secondary antibodies and imaged. The membranes were first probed with a polyclonal anti-arrestin antibody (1:1000, PA1-732; Affinity BioReagents) diluted in Odyssey blocking buffer containing $0.01 \%(\mathrm{v} / \mathrm{v})$ Tween 20 , washed four times with PBS containing $0.01 \%(\mathrm{v} / \mathrm{v})$ Tween 20 , and incubated with IRDye 680 goat anti-rabbit secondary antibody (1:5000; LI-COR Biosciences) in Odyssey blocking buffer containing $0.01 \%$ (v/v) Tween 20. The membranes were then washed as above, and the fluorescent signals were detected with the Odyssey Infrared Imaging System (LI-COR Biosciences) using the $700 \mathrm{~nm}$ channel.

To probe for GRK1 and $\beta$-tubulin, the same membranes were incubated with monoclonal anti-rhodopsin kinase 1a antibody (1:1000, MA1-720; Affinity BioReagents) and monoclonal anti- $\beta$ tubulin antibody (1:10,000, MA1-20246; Affinity BioReagents). Membranes were washed and incubated with IRDye $800 \mathrm{CW}$ goat anti-mouse secondary antibody (LI-COR BioSciences), and the fluorescent signals were detected using the $800 \mathrm{~nm}$ channel.

To quantify $\operatorname{PDE} \alpha \beta$ and $\operatorname{PDE} \gamma$, the membranes were probed with a mixture of anti-PDE6 (MOE, 1:4000; CytoSignal) and anti- $\beta$ tubulin antibodies, washed, and then incubated with IRDye 680 goat anti-rabbit and IRDye $800 \mathrm{CW}$ goat anti-mouse secondary antibodies.

RGS9-1 expression level was determined by probing the membrane with a mixture of G4695 (anti-RGS9c; 1:4000; generous gift from Dr. Ted Wensel, Baylor College of Medicine, Houston, TX) and anti- $\beta$ tubulin antibodies, washed, and incubated with IRDye $800 \mathrm{CW}$ donkey anti-goat and IRDye680 donkey anti-mouse secondary antibodies. All blocking and incubation steps were for $1 \mathrm{~h}$ at room temperature. Odyssey 2.1 software was used to quantify protein expression.

To control for loading, the protein signals (integrated fluorescence intensity) of each sample were normalized to the $\beta$-tubulin signal of the sample. The corrected integrated intensity values of the $\mathrm{Arrl}^{+/-}$, $G R K 1^{+/-}$, and $G R K 1^{+/-} A r r 1^{+/-}$samples were then normalized to the corrected integrated intensity value of the wild-type sample on the same membrane to determine the relative protein expression (Fig. 2). Each sample was run in duplicate on a different membrane and often in two different dilutions. For each genetic background, the concentrations of arrestin 1 and GRK1 determined from the two dilutions were within $20 \%$, with no evidence for systematic bias.

We did not measure expression levels of recoverin (Rv), which could control GRK1 availability. Previous work showed that $\mathrm{Arr} 1^{+/-}$mice had normal Rv expression levels (Xu et al., 1997). Furthermore, the effect of lowering $\mathrm{Rv}$ on the single-photon response differs substantially from the effects observed here, because the responses of rods from $R v^{-1-}$ and wild-type mice diverge only well after the responses have reached their maximal amplitude (Makino et al., 2004; Sampath et al., 2005).

Statistics. Reported $p$ values were based on two-sample $t$ tests.

\section{Results}

Competition between arrestin1 and GRK1 controls kinetics of rhodopsin shutoff

Figure $1 B$ illustrates the arrestin competition hypothesis in the context of a kinetic model for a single phosphorylation event.
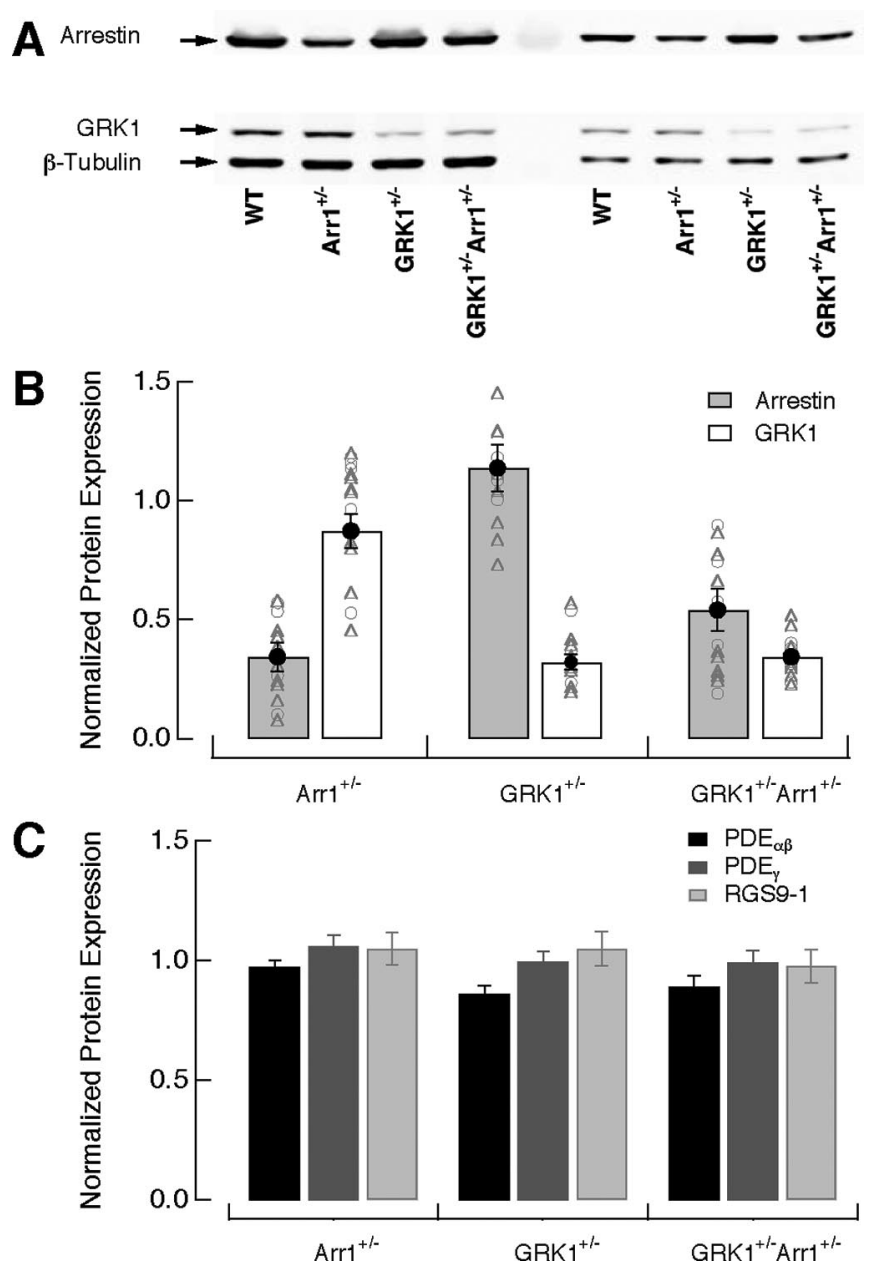

Figure 2. Protein expression in wild-type, $\mathrm{Arr}^{+/-}, \mathrm{GRK1^{+/- }}$, and $\mathrm{GRK1^{+/- }} \mathrm{Arr}^{+/-}$ rods. $A$, Representative immunoblot analysis of arrestin and GRK1 expression in retinas of wildtype and heterozygous knock-out mice. The blot was probed with polyclonal anti-arrestin, monoclonal anti-rhodopsin kinase $1 \mathrm{a}$, and monoclonal anti- $\beta$ tubulin antibodies. Two different dilutions of the same samples were run on the same blot. $\boldsymbol{B}$, Levels of arrestin1 and GRK1 expression in heterozygous knock-out retinas normalized to the levels in wild-type retinas. Integrated fluorescence intensity values were used for all analyses. Variations in sample loading were corrected for by normalizing the intensity value of each protein band to the intensity value of $\beta$-tubulin in the same lane. Open circles and triangles show the results from two different dilutions. Normalized ratios of arrestin1 and GRK1 expression compared with wild-type values for Arr $1^{+/-}$were $0.34 \pm 0.06$ and $0.87 \pm 0.07$ (mean \pm SEM; $n=5$ ), $1.14 \pm 0.10$ and $0.32 \pm 0.03$ for $G R K 1^{+/-}(n=4)$, and $0.54 \pm 0.09$ and $0.34 \pm 0.03$ for $G R K 1^{+/-}$Arr $^{+/-}$ $(n=5)$. C, Levels of PDE $\alpha \beta$, PDE $\gamma$, and RGS9-1 expression in heterozygous retinas normalized to the levels in wild-type retinas. Normalized ratios of PDE $\alpha \beta, \operatorname{PDE} \gamma$, and RGS9-1 compared with wild-type values for $A r r 1^{+/-}$were $0.97 \pm 0.03,1.06 \pm 0.05$, and $1.05 \pm 0.07(n=4)$, $0.86 \pm 0.03,0.99 \pm 0.04$, and $1.05 \pm 0.07$ for GRK1 $1^{+/-}(n=4)$, and $0.89 \pm 0.05,0.99 \pm$ 0.05 , and $0.98 \pm 0.07$ for $G R K 1^{+/-} \operatorname{Arr}^{+/-}(n=4)$.

Phosphorylation at a minimum involves two steps: GRK1 binding to active rhodopsin (reaction 2) and the subsequent attachment of a phosphate (reaction 3). For simplicity, we assume that GRK1 can only dissociate after phosphate attachment (i.e., we consider only the effective GRK1 binding rate: the product of the true binding rate and the fraction of binding events that lead to phosphate attachment). The effective GRK1 binding rate depends on the fraction of time rhodopsin is available. Our hypothesis is that, in addition to quenching rhodopsin activity, the binding of arrestin 1 to rhodopsin (reaction 1) controls rhodopsin availability and hence the effective GRK1 binding rate. 
The two roles of arrestin depicted in Figure $1 B$-rhodopsin quenching and competition with GRK1-will have opposite effects on the active lifetime of rhodopsin when the arrestin 1 concentration is altered. In the absence of any changes in quenching, the arrestin competition hypothesis predicts that reducing the arrestin 1 concentration will bias the competition in favor of GRK1, accelerating phosphorylation and $\mathrm{Rh}^{\star}$ shutoff (Fig. 1C) (for simulation details, see figure legend). In the absence of competition, the role of arrestin in quenching the activity of rhodopsin (reaction 4) means that lowering the arrestin 1 concentration will prolong the active lifetime of $\mathrm{Rh}^{\star}$ (Fig. 1D). Which of these opposing effects dominates depends on the rate constants describing each of the events depicted in Figure $1 B$. For example, if phosphate attachment (reaction 3) is much slower than GRK1 binding, then altering the binding rate through arrestin competition should have only a small effect on the overall kinetics of rhodopsin inactivation.

\section{$\mathrm{GRK}^{+/-}$and $\mathrm{GRK} 1^{+/-} \mathrm{Arr} 1^{+/-}$rods reveal strong competition between arrestin 1 and GRK1}

Arrestin competition should be prominent in rods expressing a lowered GRK1 concentration $\left(G R K 1^{+/-}\right.$rods). Lowering the GRK1 concentration should slow GRK1 binding to rhodopsin, prolonging the active lifetime of rhodopsin and increasing the sensitivity of the response kinetics to modulation of the GRK1 binding rate. Thus, we start by characterizing the response properties of these rods.

Quantitative Western blots showed that $G R K 1^{+/-}$rods contained approximately one-third as much GRK1 as wild-type rods, whereas the concentrations of arrestin 1 and other key transduction cascade components were near normal (Fig. 2). Figure $3 \mathrm{~A}$ compares estimated single-photon responses (the average response to a dim flash divided by the flash strength) from wildtype and $G R K 1^{+/-}$rods. These measurements were all made at $30^{\circ} \mathrm{C}$, a temperature at which we could also study variability in the single-photon response (see below). As expected for slower GRK1 binding, decreasing the GRK1 concentration increased the amplitude and slowed the single-photon response (Fig. $3 A$, Table $1)$. We quantified the change in kinetics by computing the integration time: the area of the response divided by its peak amplitude. Changes in amplitude $(p<0.001)$ and integration time $(p<0.0001)$ were both significant.

The changes in the single-photon response in $G R K 1^{+/-}$rods are consistent with the expected prolongation of the catalytic activity of rhodopsin. To confirm this, we used the Pepperberg analysis to quantify the decay rate of light-activated PDE activity (Pepperberg et al., 1992). Decay of PDE activity depends on the inactivation of both rhodopsin and the G-protein transducin (Fig. 1A); the decay rate for PDE activity will reflect primarily the slower of these two inactivation processes. The paradigm involves delivering bright flashes that activate PDE sufficiently strongly to minimize the internal cGMP concentration and suppress all of the circulating dark current of the rod. This manipulation should also reduce the internal calcium concentration to a minimal level, causing a constant (and high) rate of cGMP synthesis. The time the response remains in saturation then reflects the time required
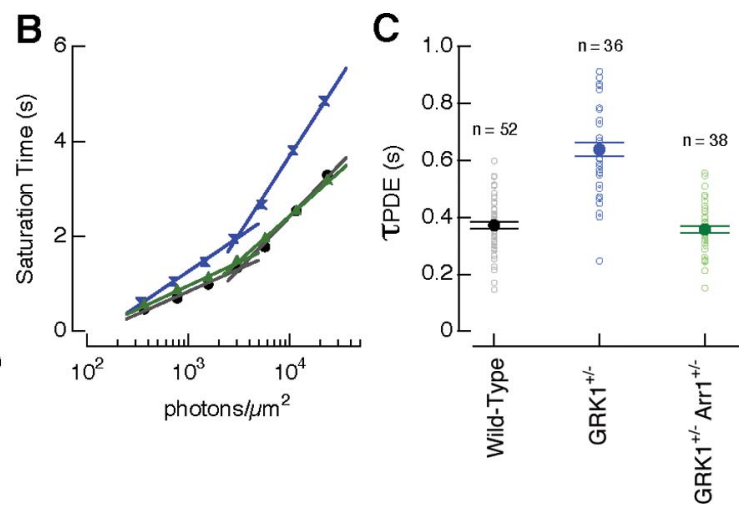

\section{$\frac{1}{\frac{1}{4}}$}

Figure 3. $\mathrm{Rh}^{*}$ inactivation depends on the relative concentration of arrestin1 and GRK1. $A$, Mean estimated single-photon

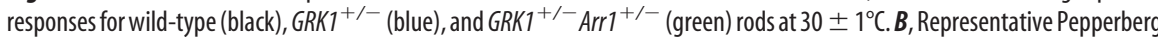
plot (time spent in saturation as a function of the log of flash intensity) for wild-type, GRK1 ${ }^{+/-}$, and $G R K 1^{+/-} A r r 1^{+/-}$rods. PDE $(n=36)$, and $360 \pm 10 \mathrm{~ms}$ for $G R K 1^{+/-} \operatorname{Arr}^{+/-} \operatorname{rods}(n=38)$.

for the PDE activity to drop to a critical value such that the cGMP synthesis rate exceeds the hydrolysis rate. Assuming that the PDE activity decays with an exponential time course, the slope of the relationship between time in saturation and the logarithm of the flash strength yields an estimate of the time constant for the decay of PDE activity (Pepperberg et al., 1992).

Figure $3 B$ plots the dependence of saturation time on flash strength for single wild-type and GRK1 $1^{+/-}$rods. The saturation time for the wild-type rod increased less steeply than that for the $G R K 1^{+/-}$rod across a wide range of flash strengths, reflecting a shorter time constant for PDE activity decay. Typical of such experiments, the relationship between saturation time and flash strength became steeper at high flash strengths. Because our interest is in the events shaping responses to dim lights, we estimated the time constant for the decay of PDE activity $\left(\tau_{\mathrm{PDE}}\right)$ from the linear region of the plot at low flash strengths. Figure $3 C$ collects results for multiple rods; $\tau_{\mathrm{PDE}}$ for wild-type rods was considerably smaller than that for $G R K 1^{+/-}$rods $(p<0.0001)$ (Fig. 3C).

Figure $3 A-C$ indicates that GRK1 binding to rhodopsin contributes substantially to the decay rate of PDE activity in $G R K 1^{+/-}$rods. In this case, the arrestin competition hypothesis (Fig. $1 B$ ) predicts that lowering the arrestin1 concentration should speed GRK1 binding to rhodopsin, accelerating PDE shutoff and producing a smaller and more rapid flash response. We used $G R K 1^{+/-} \mathrm{Arrl}^{+/-}$rods to test this prediction. GRK1 ${ }^{+/-}$ $A r r 1^{+/-}$rods expressed approximately one-third as much GRK1 and approximately one-half as much arrestin 1 as wild-type rods, whereas the concentrations of other key transduction cascade components were near normal (Fig. 2). In agreement with the arrestin competition model, GRK1 $1^{+/-} \mathrm{Arrl}^{+/-}$rods produced smaller and briefer single-photon responses than $G R K 1^{+/-}$rods (Fig. $3 A$, Table 1) $\left(p<0.01\right.$ for difference in amplitude and $p<10^{-5}$ for difference in integration time). Furthermore, the PDE activity decay time constant measured from the Pepperberg plot was considerably smaller than that of $G R K 1^{+/-}$rods (Fig. $\left.3 B, C\right)(p<0.0001)$.

In principle, arrestin competition could also regulate the rate of activation of transducin because transducin competes with arrestin1 and GRK1 for active rhodopsin. Such an effect could alter the initial rising phase of the light response. However, responses $G R K 1^{+/-}$and $G R K 1^{+/-} A r r 1^{+/-}$rods to both dim and bright flashes followed near identical initial trajectories. Two issues may contribute to a lack of an apparent effect on the initial 
A

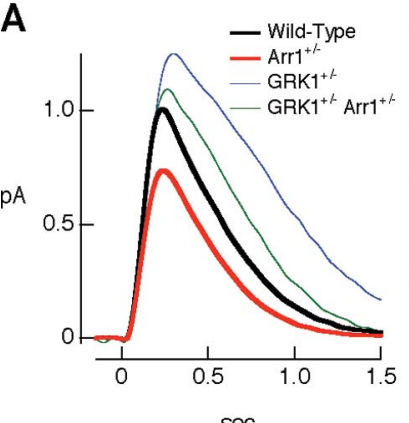

$\sec$
B

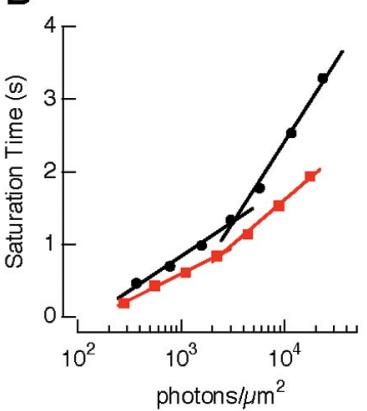

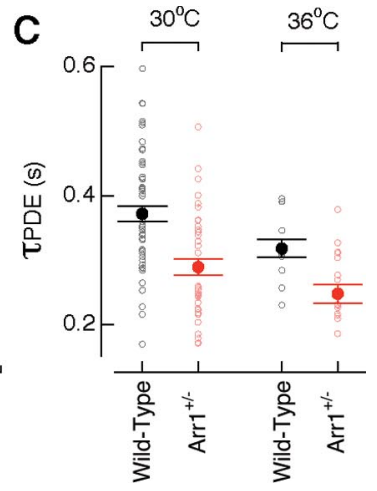

$\mathrm{Arrl}^{+/-}$rod increased less steeply with flash strength than those of the wild-type rod. Figure $4 C$ shows that $A r r 1^{+/-}$rods had a shorter $\tau_{\mathrm{PDE}}$ than wild-type rods at $30^{\circ} \mathrm{C}(p<0.0001)$ and at $36^{\circ} \mathrm{C}(p<$ 0.05 ). The reduction in response amplitude, the faster recovery phase, and the shorter $\tau_{\mathrm{PDE}}$ in the Arr1 ${ }^{+/-}$rods are consistent with more rapid rhodopsin shutoff resulting from decreased competition by arrestin 1 as depicted in Figure 1, $B$ and C. Thus, lowering the arrestin 1 concentration apparently increases the fraction of time rhodopsin is available for GRK1 binding and by doing so speeds phosphorylation and rhodopsin inactivation. This competition persists at physiological temperatures.

The decay of light-activated PDE activity reflects the inactivation kinetics of both rhodopsin and transducin. In particular, $\tau_{\mathrm{PDE}}$ depends primarily on the

Table 2. Arrestin competition model can capture the changes in response area

\begin{tabular}{lll}
\hline Strain & $\begin{array}{l}\text { Area/Area } \\
\text { experiment }\end{array}$ & $\begin{array}{l}\text { Area/Area } \\
\text { model }\end{array}$ \\
\hline GRK1 $^{+/-}(n=43)$ & $1.95 \pm 0.08$ & 2.02 \\
GRK1 $^{+/-}$Arr1 $^{+/-}(n=39)$ & $1.30 \pm 0.07$ & 1.34 \\
Arr1 $^{+/-}(n=36)$ & $0.68 \pm 0.06$ & 0.74 \\
\hline
\end{tabular}

Minimizing the mean-square error between experimental values (middle column) and predicted values (right column) yielded $\beta_{0} / \alpha=6$ and $\gamma_{0} / \sigma=8$. Experimental data are from dim flash responses.

rate of transducin activation. First, GDP-GTP exchange may be slower than the rate of binding of transducin to rhodopsin. Second, arrestin competition likely increases in strength as rhodop$\sin$ is phosphorylated and the affinity of the interaction between arrestin and rhodopsin increases (Wilden, 1995; Gibson et al., 2000; Gurevich and Gurevich, 2004; Vishnivetskiy et al., 2007).

The results of Figure $3 A-C$ indicate that the primary effect of halving the arrestin1 concentration was to increase the GRK1 binding rate rather than to slow $\mathrm{Rh}^{\star}$ quenching. This suggests that arrestin competition, at least in the presence of reduced GRK1, can play an important role in controlling the kinetics of rhodopsin shutoff.

\section{Arrestin competition reduces the catalytic activity of $R h^{*}$ in wild-type rods}

The above experiments revealed arrestin competition in rods with slowed GRK1 binding to rhodopsin. Does competition between arrestin1 and GRK1 continue at normal GRK1 concentrations, and, if so, what impact can it have on rhodopsin shutoff? Does arrestin competition persist at physiological temperatures? To answer these questions, we compared the single-photon responses and the rate of decay of PDE activity of $\mathrm{Arrl}^{+/-}$and wild-type rods at both $30^{\circ} \mathrm{C}$ and $36^{\circ} \mathrm{C}$.

$\mathrm{Arrl}^{+/-}$rods expressed approximately one-third as much arrestin 1 as wild-type rods; expression levels of GRK1 and other key transduction cascade components were near normal (Fig. 2). Figure $4 A$ compares estimated single-photon responses of wild-type and $\mathrm{Arr} 1^{+/-}$rods. Arr $1^{+/-}$rods generated smaller $(p<0.001$ for $30^{\circ} \mathrm{C}$ and $p<0.0001$ for $\left.36^{\circ} \mathrm{C}\right)$ and briefer $\left(p<0.05\right.$ for $\left.36^{\circ} \mathrm{C}\right)$ single-photon responses than wild-type rods (Table 1). Figure $4 B$ plots the dependence of saturation time on flash strength for single wild-type and $\mathrm{Arrl}^{+/-}$rods. Saturation times for the slower of these inactivation processes. Prolonging or maintaining $\tau_{\mathrm{PDE}}$ does not define which reaction is normally the rate-limiting step, but shortening $\tau_{\mathrm{PDE}}$ does (Kennedy et al., 2003; Krispel et al., 2006). Thus, the reduced $\tau_{\mathrm{PDE}}$ in $\mathrm{Arrl}^{+/-}$rods indicates that the active lifetime of rhodopsin is relatively long in wild-type rods under the conditions of our experiments. We return to this point below in the context of how variability in rhodopsin shutoff produces variability in the single-photon response.

Relative rate constants of events controlling rhodopsin inactivation To provide a quantitative description of how competition between arrestin 1 and GRK1 influences rhodopsin inactivation, we used our experimental data to estimate the average rate constants controlling steps $1-3$ in the kinetic scheme of Figure $1 B$. This is the simplest kinetic scheme consistent with the data of Figures 3 and 4 . We were particularly interested in two issues. (1) How effectively does arrestin 1 compete with GRK1 for $\mathrm{Rh}^{\star}$ binding sites? (2) What are the relative rate constants of GRK1 binding and phosphate attachment, the two events involved in a single phosphorylation cycle (Fig. $1 B$, completion of step 3 )? To answer these questions, we described reactions $1-3$ in Figure $1 B$ as a set of differential equations that depend on $\alpha, \beta, \gamma$, and $\sigma$. The fraction of time rhodopsin spends bound to arrestin1 in the model is $\beta /(\beta+\alpha)$, and the fraction of a phosphorylation cycle consumed by GRK1 binding is $\gamma /(\gamma+\sigma)$. Thus, we identified the relative rate constants for arrestin 1 association and dissociation $(\beta / \alpha)$ and for GRK1 binding and phosphate attachment $(\gamma / \sigma)$ most consistent with experiment (for details, see Materials and Methods).

We made three assumptions to compare the kinetic model to experimental data. First, we assumed that $\beta$ scaled linearly with the arrestin1 concentration $\left(\beta=\beta_{0}[\operatorname{Arr} 1] /[\operatorname{Arr} 1]_{\mathrm{WT}}\right)$ and that $\gamma$ scaled linearly with the GRK1 concentration $\left(\gamma=\gamma_{0}\right.$ [GRK1]/ $\left.[\mathrm{GRK} 1]_{\mathrm{WT}}\right)$. Concentrations of arrestin1 and GRK1 relative to wild-type rods were taken from quantitative protein analysis (Fig. $2 B$ ) (for a discussion of this assumption, see Materials and Methods). Second, we focused on the effective GRK1 binding rate, i.e., we did not include a reverse rate constant in reaction 2 of Figure $1 B$. Third, we assumed that the integral of the single-photon response (response area) was proportional to the time required for a single phosphorylation step. This assumption is reasonable 
if the active lifetime of rhodopsin is proportional to the time required for each phosphorylation event, and the transduction cascade acts linearly to convert the activity of rhodopsin to a change in current. Based on the above assumptions, we determined the ratios of $\beta_{0} / \alpha$ and $\gamma_{0} / \sigma$ that best fit the integrals of the singlephoton responses in $\mathrm{Arr1} 1^{+/-}, \mathrm{GRK1^{+/- }}$, and $\mathrm{GRK1}^{+/-} \mathrm{Arrl}^{+/-}$rods (Table 2). Because of the third assumption, the estimated ratios represent average values across different phosphorylation events (i.e., unphosphorylated rhodopsin, singly phosphorylated rhodopsin, etc). The fitting procedure is not ensured of providing a close correspondence between model and experiment because the model has two free parameters $\left(\beta_{0} / \alpha\right.$ and $\left.\gamma_{0} / \sigma\right)$ and is fit to experimentally determined values (the response areas) of each of the three mutants relative to wild type. Nonetheless, the model accounted for the measured response areas within the experimental accuracy (Table 2).

Ratios of $\beta_{0} / \alpha \sim 6$ and $\gamma_{0} / \sigma \sim 8$ minimized the mean-square error between model and experiment. These rate constants make two predictions about the phosphorylation process in wild-type rods $\left(\beta=\beta_{0}\right.$ and $\left.\gamma=\gamma_{0}\right)$ under the conditions of our experiments. First, active rhodopsin spends $\sim 85 \%$ of its time bound to arrestin 1 (Fig. $1 B$, reaction 1 ), and only $\sim 15 \%$ of the time is available for GRK1 binding. The large fraction of time rhodopsin spends interacting with arrestin 1 is a requirement for arrestin competition to control the effective GRK1 binding rate. Second, GRK1 binding is rapid compared with phosphate attachment $\left(\gamma_{0}>\sigma\right)$. This latter observation can explain why arrestin competition was revealed more robustly when GRK1 binding was slowed by reducing the GRK1 concentration.

\section{Implications of arrestin competition for single-photon response variability}

The single-photon responses of rod photoreceptors show much less trial-to-trial variability than other signals generated by single molecules (Baylor et al., 1979), such as the charge flowing through an ion channel during a single opening or the signal generated by the binding of an odorant molecule to its cognate GPCR (Bhandawat et al., 2005). Several results indicate that variability in rhodopsin shutoff rather than downstream components of the phototransduction cascade dominates variability in the single-photon response (Rieke and Baylor, 1998; Doan et al., 2006). The model most consistent with experimental observations is that $\mathrm{Rh}^{\star}$ shuts off through a series of steps (Rieke and Baylor, 1998; Field and Rieke, 2002a; Hamer et al., 2003; Doan et al., 2006; Bisegna et al., 2008). One salient aspect of the measured responses is that most of the variability in the single-photon response occurs well after the response reaches peak (Rieke and Baylor, 1998; Field and Rieke, 2002a; Hamer et al., 2003). This late variance is inconsistent with a short $\mathrm{Rh}^{\star}$ lifetime (Rieke and Baylor, 1998; Hamer et al., 2003; Krispel et al., 2006), which should cause the responses to vary in amplitude but not in shape.

The low and late variability of the single-photon responses are signatures of the underlying molecular events regulating $\mathrm{Rh}^{\star} \mathrm{ac}$ tivity (Field and Rieke, 2002a; Hamer et al., 2003). We used these characteristics in the context of the arrestin competition hypothesis to test how altering the time constants of known events in $\mathrm{Rh}^{*}$ shutoff affects reproducibility and to resolve the apparent conflict between the late time-dependent variance and the short $\mathrm{Rh}^{\star}$ lifetime reported previously (Krispel et al., 2006). The experiments and analyses described below indicate that, under the conditions of our experiments, arrestin competition tunes the kinetics of rhodopsin shutoff to minimize variability and that the active lifetime of rhodopsin persists through much of the single-photon response. 
A
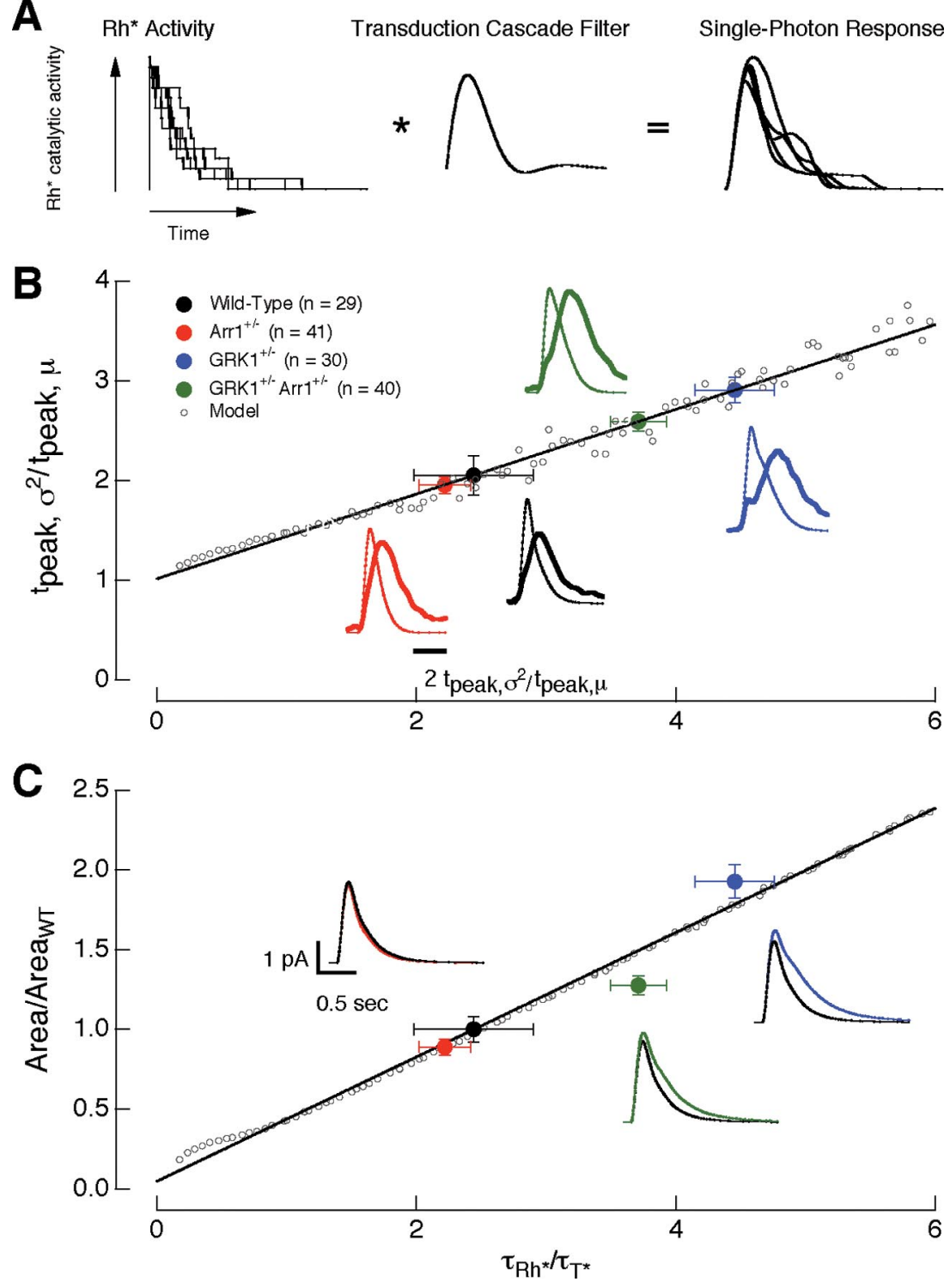

Figure 6. The lifetime of rhodopsin determines the variability and area of the single-photon responses. $\boldsymbol{A}$, Multistep shutoff model of the activity of rhodopsin. $\boldsymbol{B}$, The ratio of the time-to-peak of the time-dependent variance $\left(t_{\text {peak, } \sigma^{2}}\right)$ to the time-to-peak of the mean $\left(t_{\text {peak }, \mu}\right)$ of the single-photon responses depends on the lifetime of rhodopsin $\left(\tau_{\mathrm{Rh}}^{*}\right)$. The solid black line is fit to the predicted changes (open circles) in $t_{\text {peak }, \sigma^{2} / t_{\text {peak }, \mu}}$ as a function of $\tau_{\mathrm{Rh}}{ }^{*} \tau_{\mathrm{T}}^{*}$ was kept constant at $200 \mathrm{~ms}$. The experimental points (filled circles) are located on the fit at a $y$-axis location according to the measured $t_{\text {peak }, \sigma^{2} / t_{\text {peak }, \mu}}$ ratio; the resulting $x$-axis position estimates $\tau_{\mathrm{Rh}}{ }^{*} \boldsymbol{C}, \tau_{\mathrm{Rh}}^{*}$ controls the area of the single-photon response. Filled circles are the experimentally determined normalized response areas. These values were positioned on the $\boldsymbol{x}$-axis using the predicted $\tau_{\mathrm{Rh}}^{*}$ determined in $\boldsymbol{B}$. The solid black line is fit to the predicted changes in response area as a function of $\tau_{R h}^{*}$. Insets show the average single-photon response of 1000 simulated trials using the $\tau_{\mathrm{Rh}}^{*}$ predicted from the model for each recorded mouse strain. The model captures the relative differences in amplitude and duration of the isolated single-photon responses in wild-type and mutant rods. Error bars are SEM.

Reproducibility of the single-photon responses depends on multiple timely shutoff steps

The left panels in Figure 5A-C superimpose 10 isolated singlephoton responses from wild-type, $A r r 1^{+/-}, G R K 1^{+/-}$, and $\mathrm{GRK}^{+/-} \mathrm{Arrl}^{+/-}$rods. We isolated single-photon responses at $30^{\circ} \mathrm{C}$ because this minimized contamination from responses to 0 or multiple photons; response variability in wild-type rods was quantitatively similar at $30^{\circ} \mathrm{C}$ and $36^{\circ} \mathrm{C}$ (Doan et al., 2006). We characterized the single-photon response variability by measuring the coefficient of variation of the response areas $\left(\mathrm{CV}_{\text {area }}\right)$ ( $\mathrm{SD}$ of the areas divided by the mean area). The $\mathrm{CV}_{\text {area }}$ captures the total variability, independent of the kinetics of rhodopsin shutoff relative to the kinetics of downstream components of the transduction cascade (Field and Rieke, 2002a; Hamer et al., 2003; Doan et al., 2006). The variance attributable to the single-photon response was measured from the difference between the variance of the areas of isolated singlephoton responses and equivalent sections of dark record.

The single-photon responses of $A r r 1^{+/-}$, $G R K 1^{+/-}$, and $G R K 1^{+/-} A r r 1^{+/-}$rods all varied significantly more than those of wildtype rods (Table 1). Thus, manipulations that slow rhodopsin shutoff (via slowed GRK1 binding to rhodopsin in $G R K 1^{+/-}$ rods) as well as those that speed rhodopsin shutoff (via more rapid GRK1 binding attributable to decreased competition between arrestin 1 and GRK1 in Arr $1^{+/-}$rods) increase variability. Surprisingly, decreasing both arrestin1 and GRK1 concentrations $\left(G R K 1^{+/-} \mathrm{Arrl}^{+/-}\right.$rods) increased variability, although the single-photon response kinetics and PDE activity decay rate were near normal (Table 1).

Changes in response variability can be explained in the context of the multistep shutoff model for rhodopsin inactivation. For multiple shutoff steps to be effective in reducing response variability, each step must control a similar fraction of the total $\mathrm{Rh}^{\star}$ activity (Rieke and Baylor, 1998; Field and Rieke, 2002a; Hamer et al., 2003; Doan et al., 2006). Altering the arrestin1 or GRK1 concentrations apparently imbalances these steps and increases variability (see Discussion). The increased variability in the $G R K 1^{+/-} A r r 1^{+/-}$rods indicates that the absolute, and not just the relative, concentrations of GRK1 and arrestin 1 are critical to the generation of reproducible single-photon responses.

The late response variability is consistent with slow rhodopsin shutoff

We characterized the time course of single-photon response variability by comparing the time-dependent variance of isolated single-photon responses with the square of the mean response (Fig. $5 A-C$, right). For responses of wild-type rods, the variance $\left(\sigma^{2}\right)$ took approximately twice as long to reach its maximum as the mean response

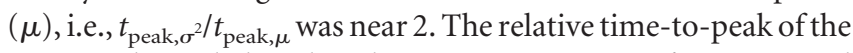
variance depended on the relative concentrations of arrestin 1 and

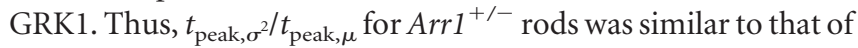
wild-type rods (Fig. $5 A$, Table 1), whereas $t_{\text {peak }, \sigma^{2} / t_{\text {peak }, \mu} \text { for both }}$ $G R K 1^{+/-}$and $G R K 1^{+/-} A r r 1^{+/-}$rods was significantly larger than 2 (Fig. $5 B, C$, Table 1). $t_{\text {peak }, \sigma^{2} / t_{\text {peak }, \mu} \text { for } G R K 1^{+/-} A r r 1^{+/-}}$ rods was significantly less than that for $G R K 1^{+/-}$rods.

The dependence of response variability on arrestin 1 and GRK1 concentrations, i.e., on manipulations that should be specific to rhodopsin, supports the idea that variability in rhodopsin 
shutoff rather than downstream components in phototransduction dominates variability in the single-photon response (Doan

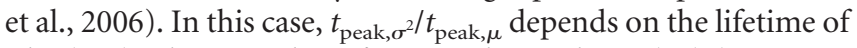
$\mathrm{Rh}^{\star}\left(\tau_{\mathrm{Rh}}^{\star}\right)$ relative to that of activated transducin $\left(\tau_{\mathrm{T}}^{\star}\right)$ (Hamer et al., 2003). If $\tau_{\mathrm{Rh}}^{\star}$ is short relative to $\tau_{\mathrm{T}}^{\star}$, then most of the variability occurs near the peak of the response because variations in $\mathrm{Rh}^{\star}$ lifetime primarily produce variations in response amplitude rather than shape. However, if $\tau_{\mathrm{Rh}}^{\star}$ is long compared with $\tau_{\mathrm{T}}^{\star}$, then most of the variability occurs during response recovery, because variations in $\mathrm{Rh}^{\star}$ lifetime alter the amplitude and duration of the single-photon responses.

To determine what constraints the measured time-dependent variances place on the ratio of the lifetimes of rhodopsin and transducin, we simulated single-photon responses by passing the modeled stochastic time course of a single rhodopsin molecule through a temporal filter that approximates the action of the phototransduction cascade (Fig. 6A) (for details, see Materials and Methods) (Rieke and Baylor, 1998; Field and Rieke, 2002a). This model assumed $\tau_{\mathrm{T}}^{\star}=200 \mathrm{~ms}$ (Krispel et al., 2006). The activity of rhodopsin was assumed to be shut off by $N$ first-order and memoryless steps attributed to rhodopsin phosphorylation and arrestin quenching. Each phosphorylation event was a twostep process (Fig. $1 B$, reactions 1-3). Arrestin quenching was modeled as a parallel step with a rate constant that increased with the number of completed phosphorylation events (Hamer et al., 2003; Vishnivetskiy et al., 2007). We used models in which an average of either 3.7 or 5.6 phosphorylation cycles completed before arrestin 1 binding (see Materials and Methods). In the first model, saturation in the transduction cascade was required to account for the low response variability. The central conclusions reached below held true for both models; we emphasize the model without saturation because it had fewer free parameters.

The multistep shutoff model was first applied to characterize the late time-to-peak of the time-dependent variance. Figure $6 B$ illustrates the predicted dependence of the relative time-to-peak of the variance $\left(t_{\text {peak, } \sigma^{2} / t_{\text {peak }, \mu}}\right)$ on the relative rhodopsin lifetime $\left(\tau_{\mathrm{Rh}}^{\star} / \tau_{\mathrm{T}}^{\star}\right)$. Consistent with the intuitive argument given above, $t_{\text {peak }, \sigma^{2}} / t_{\text {peak }, \mu}$ increased steadily as $\tau_{\mathrm{Rh}}^{\star} / \tau_{\mathrm{T}}^{\star}$ increased. We used the predictions of the model in Figure $6 B$ to estimate the relative rhodopsin lifetime for wild-type rods and rods with altered arrestin 1 and GRK1 concentrations. The experimental points (filled circles) in Figure $6 \mathrm{~B}$ are located along the line fit through the model prediction (open circles) according to the measured $t_{\text {peak }, \sigma^{2}} / t_{\text {peak }, \mu}$ ratio ( $y$-axis). The resulting $x$-axis position of the experimental points provides an estimate of the relative rhodopsin lifetime. Accounting for the late time-to-peak of the wild-type singlephoton responses required $\tau_{\mathrm{Rh}}^{\star} / \tau_{\mathrm{T}}^{\star} \approx 2.4$ (Fig. $6 \mathrm{~B}$, black circle).

To test the validity of the model, we used the rhodopsin lifetimes estimated from the time-dependent variance to predict the relative areas of the single-photon responses. The model predicts that the response area depends linearly on the lifetime of rhodop$\sin$ (Fig. 6C, open circles and line). The position of the experimental points in Figure $6 C$ (filled circles) are determined from the measured areas ( $y$-axis) and the estimated $\tau_{\mathrm{Rh}}^{\star} / \tau_{\mathrm{T}}^{\star}$ from Figure $6 B$ (x-axis). Insets show predicted single-photon responses from the model (for experimental results, see Figs. $3 A, 4 A$ ). The model, fit only to the time-to-peak of the variance, does a reasonable job accounting for the changes in response area and time course produced by alterations in arrestin 1 and GRK1 concentrations.

The analysis in Figure 6 indicates that, in the context of the multistep shutoff model, the late time-to-peak of the time- dependent variance requires that rhodopsin remain active for much of the duration of the single-photon response, i.e., that the active lifetime of rhodopsin exceeds that of transducin. A short rhodopsin lifetime would both fail to predict the time course of the time-dependent variance in wild-type rods and the change in variance produced by alterations in arrestin1 and GRK1 concentrations.

\section{Discussion}

We studied how changes in the concentration of GRK1 and arrestin 1 affected the single-photon responses of rod photoreceptors. Our experiments indicate that arrestin1 competes with GRK1 for binding sites on rhodopsin and in doing so can slow both rhodopsin inactivation and facilitate low variability of the active lifetime of single rhodopsin molecules and of the resulting single-photon responses. A similar tuning of the kinetics of phosphorylation through arrestin competition may be a general feature of how GPCR activity is controlled, particularly for GPCRs in which arrestin and kinase bind to overlapping domains of the receptor.

\section{Arrestin competition modulates single-photon response kinetics}

Arrestins play a well known role in quenching the activity of G-protein-coupled receptors (Kühn et al., 1984; Wilden, 1995; Xu et al., 1997; Makino et al., 2003; Gurevich and Gurevich, 2004; Burns et al., 2006). Our results indicate that arrestins can play a second, previously unappreciated, role in regulating receptor activity through competition with kinases. Thus, the concentration of arrestin 1 in the rod outer segment regulates the amount of time that rhodopsin is available for GRK1 binding and hence the rate of rhodopsin inactivation. Fitting the simple kinetic scheme in Figure 1 to properties of the measured responses indicated that active rhodopsin spends most of its time bound to arrestin 1 and unavailable for GRK1 binding. This competition likely is initially weak and increases as phosphates are attached to rhodopsin (Wilden, 1995; Gibson et al., 2000; Gurevich and Gurevich, 2004; Vishnivetskiy et al., 2007). Phosphorylation is also likely to enhance competition between arrestin and transducin and hence slow transducin activation. Fits to the kinetic scheme of Figure 1 indicated that GRK1 binding, even when slowed by arrestin competition, is more rapid than the subsequent phosphate attachment step.

Microcalorimetry experiments (Langlois et al., 1996) do not show a speeding of rhodopsin inactivation with lowered arrestin 1 concentrations; these experiments instead support the role of arrestin 1 in quenching the activity of rhodopsin. However, these experiments and other biochemical assays of arrestin 1 binding (Wilden et al., 1986; Wilden, 1995; Zhang et al., 1997; Brannock et al., 1999) use arrestin 1 concentrations 10-1000 times lower than physiological levels (Hamm and Bownds, 1986; Schubert et al., 1999). Under these conditions, the arrestin quenching interaction is likely to dominate the competition interaction. For example, the kinetic model in Figure $1 B$ predicts that lowering the arrestin 1 concentration 10-fold from physiological levels would increase the fraction of time rhodopsin is available for GRK1 binding from $\sim 15$ to $\sim 60 \%$. An additional twofold reduction in arrestin 1 concentration would increase this to $\sim 75 \%$, producing a $\sim 15 \%$ increase in the rate of GRK1 binding to rhodopsin. The same calculation, applied to physiological arrestin1 levels, predicts a $\sim 60 \%$ increase in the GRK1 binding rate. Thus, when the arrestin 1 concentration is substantially less than normal, the slowing of rhodopsin quenching by an additional reduction in 
arrestin 1 concentration may dominate any speeding of rhodopsin inactivation by decreased competition between arrestin 1 and GRK1.

\section{Arrestin competition minimizes variability in rhodopsin inactivation}

A long-standing and puzzling observation about the singlephoton responses of rods is their low variability (Baylor et al., 1979). This low variability requires tight regulation of the active lifetime of rhodopsin. Several lines of evidence support a model in which the activity of a single rhodopsin molecule is terminated through a series of steps or transitions (Rieke and Baylor, 1998; Field and Rieke, 2002a; Hamer et al., 2003; Doan et al., 2006). The effectiveness of this model depends on the near equal distribution of the total activity of rhodopsin among these steps, e.g., if a single step controls the majority of the activity of rhodopsin, then variability in the duration of this step will dominate variability in the effective lifetime of rhodopsin. Our results suggest that competition between arrestin 1 and GRK1 is important in producing a uniform distribution of the activity of rhodopsin across shutoff steps.

Decreasing the concentration of arrestin1 and/or GRK1 increased single-photon response variability. This suggests that the distribution of the activity of rhodopsin among steps is made less uniform when arrestin competition is altered. Indeed, we could capture the increase in variability and the change in timedependent variance for the $A r r 1^{+/-}, G R K 1^{+/-}$, and $G R K 1^{+/-}$ $A r r 1^{+/-}$rods when we altered the relative timing of the steps in the stochastic model. We did not include this model because it introduced unconstrained parameters. Mechanistically, a disruption of uniform timing of steps in rhodopsin shutoff suggests that the competition between arrestin1 and GRK1 depends on how many phosphates are already attached to the $\mathrm{C}$ terminus of rhodopsin. Consistent with this interpretation, the affinity of the interaction between arrestin 1 and rhodopsin increases as rhodopsin is phosphorylated (Wilden, 1995; Gibson et al., 2000; Gurevich and Gurevich, 2004; Vishnivetskiy et al., 2007).

The native concentrations of arrestin 1 and GRK1 are well suited to maintain low variability in rhodopsin shutoff. This adds to a perplexing functional question: why has the rod gone to such lengths to produce responses with such low variability? One suggestion is that low variability is required for accurate encoding of the time of photon absorption (Rieke and Baylor, 1998). The identification of mice with subtle changes in response variability could provide a needed tool to test this hypothesis.

\section{The activity of rhodopsin persists through much of the single-photon response}

The relative lifetimes of rhodopsin and the G-protein transducin has been a debated issue in phototransduction. Recent work has provided good evidence, at least under some experimental conditions, that the lifetime of the activated state of transducin is long compared with that of rhodopsin (Krispel et al., 2006). This issue has implications for both the site of amplification in the system and the underlying mechanism regulating the variability of the single-photon responses. A short rhodopsin lifetime implies that only $\sim 20$ transducin molecules are activated, on average, while rhodopsin is active (Krispel et al., 2006). A brief rhodopsin lifetime also predicts that variability in the activity of rhodopsin primarily alters the amplitude but not the duration of the single-photon responses. Measured single-photon responses in toad, mouse, guinea pig, and primate rods, however, vary little in amplitude but more substantially in response recovery (Rieke and Baylor, 1998; Field and Rieke, 2002a; Hamer et al., 2003). As discussed below, differences in experimental conditions appear to explain this discrepancy.

Several results reported here support a relatively long active rhodopsin lifetime. First, we found that the lifetime of lightactivated PDE decreased when the arrestin 1 concentration was decreased. Manipulations that prolong PDE decay do not define which reaction is normally the rate-limiting step, whereas manipulations that shorten PDE decay do (Kennedy et al., 2003; Krispel et al., 2006). Second, we showed that the systematic changes in the response kinetics with alterations in arrestin 1 and/or GRK1 concentration are consistent with changes in the GRK1 binding rates predicted by the arrestin competition model. Finally, we demonstrated that the conserved characteristic time-dependent variance of the single-photon responses can be explained by a long rhodopsin lifetime (Fig. 6).

We estimated that the active lifetime of rhodopsin is approximately two times longer than the previously estimated active lifetime of transducin (Krispel et al., 2006) but similar to the ratio of $\sim 1.5$ predicted from modeling by Hamer et al. (2003). The relative active lifetimes of rhodopsin and transducin appear to depend on experimental conditions (see Materials and Methods). Specifically, we found that dim flash responses measured in Locke's solution, as in the experiments of Krispel and colleagues, were faster and less sensitive than those measured in our experiments using Ames solution (supplemental Fig. 1, available at www.jneurosci.org as supplemental material). Decay of PDE activity in Locke's solution is also approximately two times faster than that in Ames solution. These differences are consistent with slower rhodopsin inactivation in Ames solution. The underlying factors contributing to these differences are not understood.

The dependence of rod responses on recording conditions is striking and unexplained. It is not clear what conditions most closely resemble those in vivo. The kinetics of rod responses measured in Locke's solution resemble the rod component of the electroretinogram (Lyubarsky and Pugh, 1996; Hetling and Pepperberg, 1999): the time-to-peak of the estimated responses to sub-saturating flashes $(\sim 110-130 \mathrm{~ms}$ for single rod responses vs $\sim 80-90 \mathrm{~ms}$ for the electroretinogram) and the rate of decay of PDE activity ( $\sim 180-250 \mathrm{~ms}$ for single rod responses vs $\sim 190-$ $230 \mathrm{~ms}$ for the electroretinogram) are similar. However, the sensitivity of rod responses recorded in Ames solution is in closer agreement with the electroretinogram (half-saturating flash strengths are $\sim 9 \mathrm{Rh}^{\star}$ for single rod responses in Ames solution, $\sim 20 \mathrm{Rh}^{\star}$ in Locke's solution, and $\sim 10 \mathrm{Rh}^{\star}$ for the electroretinogram) (see supplemental Materials, available at www.jneurosci. org as supplemental material) (Hetling and Pepperberg, 1999). Furthermore, the isolated rod component of the electroretinogram shows a prominent contribution from the rod inner segment (Green and Kapousta-Bruneau, 1999; Nymark et al., 2005; Kang Derwent et al., 2007), complicating comparison with outer segment transduction currents. Ames and Locke's solutions are of course only two possibilities, and it is likely that in vivo conditions differ from both. Resolving this issue will be an important aspect of future work.

\section{Regulating the concentrations of proteins involved in receptor shutoff as a mechanism to control GPCR-mediated signals}

Controlling the relative concentrations of critical regulatory factors is a common approach used by biological systems to modulate cellular activities, including cellular differentiation, cell cycle checkpoints, organ development, inflammation, and chemotaxis 
(Parent and Devreotes, 1999; Ashe and Briscoe, 2006; Charest and Firtel, 2006; Métayé et al., 2006; Pinsky et al., 2006; Vroon et al., 2006). Our finding that arrestin competition has profound effects on the kinetics and variability of rhodopsin inactivation provides additional evidence for this mechanism in GPCR systems (Detwiler et al., 2000; Wang et al., 2004). Specifically, the observation that changes in the arrestin 1 concentration in dark-adapted rods result in altered and variable single-photon responses suggests that arrestin 1 translocation yields an appropriate concentration of arrestin 1 in the outer segment to compete with GRK1 and enable reliable signal transduction.

In other GPCR-mediated systems, such as the heart or the immune system, minute and prolonged perturbations in GRK concentrations are pathological (Hansen et al., 2006; Métayé et al., 2006; Vroon et al., 2006). An increase in GRK2 is associated with congestive heart failure and hypertension, whereas decreases in GRK2, GRK3, and GRK6 are involved in improper inflammatory responses (Lombardi et al., 2001; Vroon et al., 2006; Lymperopoulos et al., 2007). Current therapeutic interventions, such as $\beta$-blockers in the treatment of heart failure and hypertension, directly target GPCRs. If a fundamental strategy in regulating GPCR activity is through a careful balance of arrestin and GRK concentrations, then tissue-specific modulations of GRK and/or arrestin activity might be a new and useful therapeutic intervention for GPCR-related diseases.

\section{References}

Ashe HL, Briscoe J (2006) The interpretation of morphogen gradients. Development 133:385-394.

Baylor DA, Lamb TD, Yau KW (1979) Responses of retinal rods to single photons. J Physiol 288:613-634.

Bhandawat V, Reisert J, Yau KW (2005) Elementary response of olfactory receptor neurons to odorants. Science 308:1931-1934.

Bisegna P, Caruso G, Andreucci D, Shen L, Gurevich VV, Hamm HE, DiBenedetto E (2008) Diffusion of the second messengers in the cytoplasm acts as a variability suppressor of the single photon response in vertebrate phototransduction. Biophys J 94:3363-3383.

Brannock MT, Weng K, Robinson PR (1999) Rhodopsin's carboxylterminal threonines are required for wild-type arrestin-mediated quench of transducin activation in vitro. Biochemistry 38:3770-3777.

Broekhuyse RM, Tolhuizen EF, Janssen AP, Winkens HJ (1985) Light induced shift and binding of s-antigen in retinal rods. Curr Eye Res $4: 613-618$

Buck LB (2000) The molecular architecture of odor and pheromone sensing in mammals. Cell 100:611-618.

Burns ME, Mendez A, Chen J, Baylor DA (2002) Dynamics of cyclic GMP synthesis in retinal rods. Neuron 36:81-91.

Burns ME, Mendez A, Chen CK, Almuete A, Quillinan N, Simon MI, Baylor DA, Chen J (2006) Deactivation of phosphorylated and nonphosphorylated rhodopsin by arrestin splice variants. J Neurosci 26:1036-1044.

Calvert PD, Govardovskii VI, Krasnoperova N, Anderson RE, Lem J, Makino CL (2001) Membrane protein diffusion sets the speed of rod phototransduction. Nature 411:90-94.

Chandrashekar J, Hoon MA, Ryba NJ, Zuker CS (2006) The receptors and cells for mammalian taste. Nature 444:288-294.

Charest PG, Firtel RA (2006) Feedback signaling controls leading-edge formation during chemotaxis. Curr Opin Genet Dev 16:339-347.

Chen CK, Burns ME, Spencer M, Niemi GA, Chen J, Hurley JB, Baylor DA, Simon MI (1999) Abnormal photoresponses and light-induced apoptosis in rods lacking rhodopsin kinase. Proc Natl Acad Sci U S A 96:3718-3722.

Chen J, Makino CL, Peachey NS, Baylor DA, Simon MI (1995) Mechanisms of rhodopsin inactivation in vivo as revealed by a $\mathrm{COOH}$-terminal truncation mutant. Science 267:374-377.

Detwiler PB, Ramanathan S, Sengupta A, Shraiman BI (2000) Engineering aspects of enzymatic signal transduction: photoreceptors in the retina. Biophys J 79:2801-2817.

Doan T, Mendez A, Detwiler PB, Chen J, Rieke F (2006) Multiple phosphor- ylation sites confer reproducibility of the rod's single-photon responses. Science 313:530-533.

Eglen RM, Bosse R, Reisine T (2007) Emerging concepts of guanine nucleotide-binding protein-coupled receptor (GPCR) function and implications for high throughput screening. Assay Drug Dev Technol 5:425-451.

Field GD, Rieke F (2002a) Mechanisms regulating variability of the single photon responses of mammalian rod photoreceptors. Neuron 35:733-747.

Field GD, Rieke F (2002b) Nonlinear signal transfer from mouse rods to bipolar cells and implications for visual sensitivity. Neuron 34:773-785.

Field GD, Sampath AP, Rieke F (2005) Retinal processing near absolute threshold: from behavior to mechanism. Annu Rev Physiol 67:491-514.

Gibson SK, Parkes JH, Liebman PA (2000) Phosphorylation modulates the affinity of light-activated rhodopsin for $\mathrm{G}$ protein and arrestin. Biochemistry 39:5738-5749.

Green DG, Kapousta-Bruneau NV (1999) A dissection of the electroretinogram from the isolated rat retina with microelectrodes and drugs. Vis Neurosci 16:727-741.

Gurevich VV, Benovic JL (1992) Cell-free expression of visual arrestin. Truncation mutagenesis identifies multiple domains involved in rhodopsin interaction. J Biol Chem 267:21919-21923.

Gurevich VV, Benovic JL (1993) Visual arrestin interaction with rhodopsin. Sequential multisite binding ensures strict selectivity toward lightactivated phosphorylated rhodopsin. J Biol Chem 268:11628-11638.

Gurevich VV, Benovic JL (1995) Visual arrestin binding to rhodopsin. Diverse functional roles of positively charged residues within the phosphorylation-recognition region of arrestin. J Biol Chem 270:6010-6016.

Gurevich VV, Gurevich EV (2004) The molecular acrobatics of arrestin activation. Trends Pharmacol Sci 25:105-111.

Hamer RD, Nicholas SC, Tranchina D, Liebman PA, Lamb TD (2003) Multiple steps of phosphorylation of activated rhodopsin can account for the reproducibility of vertebrate rod single-photon responses. J Gen Physiol 122:419-444.

Hamm HE, Bownds MD (1986) Protein complement of rod outer segments of frog retina. Biochemistry 25:4512-4523.

Hansen JL, Theilade J, Aplin M, Sheikh SP (2006) Role of G-proteincoupled receptor kinase 2 in the heart: do regulatory mechanisms open novel therapeutic perspectives? Trends Cardiovasc Med 16:169-177.

Hanson SM, Gurevich EV, Vishnivetskiy SA, Ahmed MR, Song X, Gurevich VV (2007a) Each rhodopsin molecule binds its own arrestin. Proc Natl Acad Sci U S A 104:3125-3128.

Hanson SM, Van Eps N, Francis DJ, Altenbach C, Vishnivetskiy SA, Arshavsky VY, Klug CS, Hubbell WL, Gurevich VV (2007b) Structure and function of the visual arrestin oligomer. EMBO J 26:1726-1736.

Hetling JR, Pepperberg DR (1999) Sensitivity and kinetics of mouse rod flash responses determined in vivo from paired-flash electroretinograms. J Physiol 516:593-609.

Imamoto Y, Tamura C, Kamikubo H, Kataoka M (2003) Concentrationdependent tetramerization of bovine visual arrestin. Biophys J 85:1186-1195.

Kang Derwent JJ, Saszik SM, Maeda H, Little DM, Pardue MT, Frishman LJ, Pepperberg DR (2007) Test of the paired-flash electroretinographic method in mice lacking b-waves. Vis Neurosci 24:141-149.

Kelleher DJ, Johnson GL (1990) Characterization of rhodopsin kinase purified from bovine rod outer segments. J Biol Chem 265:2632-2639.

Kennedy MJ, Lee KA, Niemi GA, Craven KB, Garwin GG, Saari JC, Hurley JB (2001) Multiple phosphorylation of rhodopsin and the in vivo chemistry underlying rod photoreceptor dark adaptation. Neuron 31:87-101.

Kennedy MJ, Sowa ME, Wensel TG, Hurley JB (2003) Acceleration of key reactions as a strategy to elucidate the rate-limiting chemistry underlying phototransduction inactivation. Invest Ophthalmol Vis Sci 44:1016-1022.

Kobilka BK, Deupi X (2007) Conformational complexity of G-proteincoupled receptors. Trends Pharmacol Sci 28:397-406.

Koch KW, Stryer L (1988) Highly cooperative feedback control of retinal rod guanylate cyclase by calcium ions. Nature 334:64-66.

Krispel CM, Chen D, Melling N, Chen YJ, Martemyanov KA, Quillinan N, Arshavsky VY, Wensel TG, Chen CK, Burns ME (2006) RGS expression rate-limits recovery of rod photoresponses. Neuron 51:409-416.

Kristiansen K (2004) Molecular mechanisms of ligand binding, signaling, and regulation within the superfamily of g-protein-coupled receptors: 
molecular modeling and mutagenesis approaches to receptor structure and function. Pharmacol Ther 103:21-80.

Krupnick JG, Gurevich VV, Schepers T, Hamm HE, Benovic JL (1994) Arrestin-rhodopsin interaction. Multi-site binding delineated by peptide inhibition. J Biol Chem 269:3226-3232.

Krupnick JG, Gurevich VV, Benovic JL (1997) Mechanism of quenching of phototransduction. binding competition between arrestin and transducin for phosphorhodopsin. J Biol Chem 272:18125-18131.

Kühn H, Hall SW, Wilden U (1984) Light-induced binding of 48-kDa protein to photoreceptor membranes is highly enhanced by phosphorylation of rhodopsin. FEBS Lett 176:473-478.

Langlois G, Chen CK, Palczewski K, Hurley JB, Vuong TM (1996) Responses of the phototransduction cascade to dim light. Proc Natl Acad Sci U S A 93:4677-4682.

Ling Y, Ascano M, Robinson P, Gregurick SK (2004) Experimental and computational studies of the desensitization process in the bovine rhodopsin-arrestin complex. Biophys J 86:2445-2454.

Lombardi MS, Kavelaars A, Cobelens PM, Schmidt RE, Schedlowski M, Heijnen CJ (2001) Adjuvant arthritis induces down-regulation of G proteincoupled receptor kinases in the immune system. J Immunol 166:1635-1640.

Lymperopoulos A, Rengo G, Funakoshi H, Eckhart AD, Koch WJ (2007) Adrenal GRK2 upregulation mediates sympathetic overdrive in heart failure. Nat Med 13:315-323.

Lyubarsky AL, Pugh EN Jr (1996) Recovery phase of the murine rod photoresponse reconstructed from electroretinographic recordings. J Neurosci 16:563-571.

Maeda T, Imanishi Y, Palczewski K (2003) Rhodopsin phosphorylation: 30 years later. Prog Retin Eye Res 22:417-434.

Makino CL, Wen XH, Lem J (2003) Piecing together the timetable for visual transduction with transgenic animals. Curr Opin Neurobiol 13:404-412.

Makino CL, Dodd RL, Chen J, Burns ME, Roca A, Simon MI, Baylor DA (2004) Recoverin regulates light-dependent phosphodiesterase activity in retinal rods. J Gen Physiol 123:729-741.

Mendez A, Burns ME, Roca A, Lem J, Wu LW, Simon MI, Baylor DA, Chen J (2000) Rapid and reproducible deactivation of rhodopsin requires multiple phosphorylation sites. Neuron 28:153-164.

Métayé T, Perdrisot R, Kraimps JL (2006) GRKs and arrestins: the therapeutic pathway (in French)? Med Sci (Paris) 22:537-543.

Nymark S, Heikkinen H, Haldin C, Donner K, Koskelainen A (2005) Light responses and light adaptation in rat retinal rods at different temperatures. J Physiol 567:923-938.

Ohguro H, Van Hooser JP, Milam AH, Palczewski K (1995) Rhodopsin phosphorylation and dephosphorylation in vivo. J Biol Chem 270:14259-14262.

Palczewski K, Buczyłko J, Kaplan MW, Polans AS, Crabb JW (1991) Mechanism of rhodopsin kinase activation. J Biol Chem 266:12949-12955.

Parent CA, Devreotes PN (1999) A cell's sense of direction. Science 284:765-770.

Pepperberg DR, Cornwall MC, Kahlert M, Hofmann KP, Jin J, Jones GJ, Ripps H (1992) Light-dependent delay in the falling phase of the retinal rod photoresponse. Vis Neurosci 8:9-18.

Peterson JJ, Tam BM, Moritz OL, Shelamer CL, Dugger DR, McDowell JH, Hargrave PA, Papermaster DS, Smith WC (2003) Arrestin migrates in photoreceptors in response to light: a study of arrestin localization using an arrestin-gfp fusion protein in transgenic frogs. Exp Eye Res 76:553-563.

Pinsky BA, Kotwaliwale CV, Tatsutani SY, Breed CA, Biggins S (2006) Glc7/ protein phosphatase 1 regulatory subunits can oppose the Ipl1/aurora protein kinase by redistributing glc7. Mol Cell Biol 26:2648-2660.

Premont RT, Gainetdinov RR (2007) Physiological roles of g proteincoupled receptor kinases and arrestins. Annu Rev Physiol 69:511-534.

Raman D, Osawa S, Weiss ER (1999) Binding of arrestin to cytoplasmic loop mutants of bovine rhodopsin. Biochemistry 38:5117-5123.

Raman D, Osawa S, Gurevich VV, Weiss ER (2003) The interaction with the cytoplasmic loops of rhodopsin plays a crucial role in arrestin activation and binding. J Neurochem 84:1040-1050.

Rieke F, Baylor DA (1998) Origin of reproducibility in the responses of retinal rods to single photons. Biophys J 75:1836-1857.

Sampath AP, Strissel KJ, Elias R, Arshavsky VY, McGinnis JF, Chen J, Kawamura S, Rieke F, Hurley JB (2005) Recoverin improves rodmediated vision by enhancing signal transmission in the mouse retina. Neuron 46:413-420.

Schubert C, Hirsch JA, Gurevich VV, Engelman DM, Sigler PB, Fleming KG (1999) Visual arrestin activity may be regulated by self-association. J Biol Chem 274:21186-21190.

Shi W, Osawa S, Dickerson CD, Weiss ER (1995) Rhodopsin mutants discriminate sites important for the activation of rhodopsin kinase and gt. J Biol Chem 270:2112-2119.

Tamura T, Nakatani K, Yau KW (1991) Calcium feedback and sensitivity regulation in primate rods. J Gen Physiol 98:95-130.

Vishnivetskiy SA, Paz CL, Schubert C, Hirsch JA, Sigler PB, Gurevich VV (1999) How does arrestin respond to the phosphorylated state of rhodopsin? J Biol Chem 274:11451-11454.

Vishnivetskiy SA, Raman D, Wei J, Kennedy MJ, Hurley JB, Gurevich VV (2007) Regulation of arrestin binding by rhodopsin phosphorylation level. J Biol Chem 282:32075-32083.

Vroon A, Heijnen CJ, Kavelaars A (2006) GRKs and arrestins: regulators of migration and inflammation. J Leukoc Biol 80:1214-1221.

Wang Q, Zhao J, Brady AE, Feng J, Allen PB, Lefkowitz RJ, Greengard P, Limbird LE (2004) Spinophilin blocks arrestin actions in vitro and in vivo at G protein-coupled receptors. Science 304:1940-1944.

Whitlock GG, Lamb TD (1999) Variability in the time course of single photon responses from toad rods: termination of rhodopsin's activity. Neuron 23:337-351.

Wilden U (1995) Duration and amplitude of the light-induced cGMP hydrolysis in vertebrate photoreceptors are regulated by multiple phosphorylation of rhodopsin and by arrestin binding. Biochemistry 34:1446-1454 .

Wilden U, Kühn H (1982) Light-dependent phosphorylation of rhodopsin: number of phosphorylation sites. Biochemistry 21:3014-3022.

Wilden U, Hall SW, Kühn H (1986) Phosphodiesterase activation by photoexcited rhodopsin is quenched when rhodopsin is phosphorylated and binds the intrinsic 48-kDa protein of rod outer segments. Proc Natl Acad Sci U S A 83:1174-1178.

Xu J, Dodd RL, Makino CL, Simon MI, Baylor DA, Chen J (1997) Prolonged photoresponses in transgenic mouse rods lacking arrestin. Nature 389:505-509.

Zhang H, Li S, Doan T, Rieke F, Detwiler PB, Frederick JM, Baehr W (2007) Deletion of PrBP/delta impedes transport of GRK1 and PDE6 catalytic subunits to photoreceptor outer segments. Proc Natl Acad Sci U S A 104:8857-8862.

Zhang L, Sports CD, Osawa S, Weiss ER (1997) Rhodopsin phosphorylation sites and their role in arrestin binding. J Biol Chem 272:14762-14768. 